\title{
From Private Simultaneous Messages to Zero-Information Arthur-Merlin Protocols and Back*
}

\author{
Benny Applebaum ${ }^{\dagger} \quad$ Pavel Raykov ${ }^{\dagger}$
}

\begin{abstract}
Göös, Pitassi and Watson (ITCS, 2015) have recently introduced the notion of Zero-Information Arthur-Merlin Protocols (ZAM). In this model, which can be viewed as a private version of the standard Arthur-Merlin communication complexity game, Alice and Bob are holding a pair of inputs $x$ and $y$ respectively, and Merlin, the prover, attempts to convince them that some public function $f$ evaluates to 1 on $(x, y)$. In addition to standard completeness and soundness, Göös et al., require a "zero-knowledge" property which asserts that on each yes-input, the distribution of Merlin's proof leaks no information about the inputs $(x, y)$ to an external observer.

In this paper, we relate this new notion to the well-studied model of Private Simultaneous Messages (PSM) that was originally suggested by Feige, Naor and Kilian (STOC, 1994). Roughly speaking, we show that the randomness complexity of ZAM corresponds to the communication complexity of PSM, and that the communication complexity of ZAM corresponds to the randomness complexity of PSM. This relation works in both directions where different variants of PSM are being used. As a secondary contribution, we reveal new connections between different variants of PSM protocols which we believe to be of independent interest.

Our results give rise to better ZAM protocols based on existing PSM protocols, and to better protocols for conditional disclosure of secrets (a variant of PSM) from existing ZAMs.
\end{abstract}

\section{Introduction}

In this paper we reveal an intimate connection between two seemingly unrelated models for noninteractive information-theoretic secure computation. We begin with some background.

\subsection{Zero-Information Unambiguous Arthur-Merlin Communication Protocols}

Consider a pair of computationally-unbounded (randomized) parties, Alice and Bob, each holding an $n$-bit input, $x$ and $y$ respectively, to some public function $f:\{0,1\}^{n} \times\{0,1\}^{n} \rightarrow\{0,1\}$. In our first model, a third party, Merlin, wishes to convince Alice and Bob that their joint input is mapped to 1 (i.e., $(x, y)$ is in the language $\left.f^{-1}(1)\right)$. Merlin gets to see the parties' inputs $(x, y)$ and their private randomness $r_{A}$ and $r_{B}$, and is allowed to send a single message ("proof") $p$ to both parties.

${ }^{*}$ A preliminary version of this paper appears in TCC 2016-A AR16.

${ }^{\dagger}$ School of Electrical Engineering, Tel-Aviv University, \{bennyap, pavelraykov\}@post.tau.ac.il. Supported by the European Union's Horizon 2020 Programme (ERC-StG-2014-2020) under grant agreement no. 639813 ERC-CLC, ISF grant 1155/11, GIF grant 1152/2011, and the Check Point Institute for Information Security. This work was done in part while the first author was visiting the Simons Institute for the Theory of Computing, supported by the Simons Foundation and by the DIMACS/Simons Collaboration in Cryptography through NSF grant CNS-1523467. 
Then, each party decides whether to accept the proof based on its input and its private randomness. We say that the protocol accepts $p$ if both parties accept it. The protocol is required to satisfy natural properties of (perfect) completeness and soundness. Namely, if $(x, y) \in f^{-1}(1)$ then there is always a proof $p=p\left(x, y, r_{A}, r_{B}\right)$ that is accepted by both parties, whereas if $(x, y) \in f^{-1}(0)$ then, with probability $1-\delta$ (over the coins of Alice and Bob), no such proof exists. As usual in communication-complexity games the goal is to minimize the communication complexity of the protocol, namely the length of the proof $p$.

This model, which is well studied in the communication complexity literature [BFS86, Kla03, Kla10, is viewed as the communication complexity analogue of AM protocols [BM88]. Recently, Göös, Pitassi and Watson GPW15] suggested a variant of this model which requires an additional "zero-knowledge" property defined as follows: For any 1-input $(x, y) \in f^{-1}(1)$, the proof sent by the honest prover provides no information on the inputs $(x, y)$ to an external viewer. Formally, the random variable $p_{x, y}=p\left(x, y, r_{A}, r_{B}\right)$ induced by a random choice of $r_{A}$ and $r_{B}$ should be distributed according to some universal distribution $D$ which is independent of the specific 1-input $(x, y)$. Moreover, an additional Unambiguity property is required: any 1-input $(x, y) \in f^{-1}(1)$ and any pair of strings $\left(r_{A}, r_{B}\right)$ uniquely determine a single accepting proof $p\left(x, y, r_{A}, r_{B}\right)$.

This modified version of AM protocols (denoted by ZAM) was originally presented in attempt to explain the lack of explicit nontrivial lower bounds for the communication required by AM protocols. Indeed, Göös et al., showed that any function $f:\{0,1\}^{n} \times\{0,1\}^{n} \rightarrow\{0,1\}$ admits a ZAM protocol with at most exponential communication complexity of $O\left(2^{n}\right)$. Since the transcript of a ZAM protocol carries no information on the inputs, the mere existence of such protocols forms a "barrier" against "information complexity" based arguments. This suggests that, at least in their standard form, such arguments cannot be used to prove lower bounds against AM protocols (even with Unambiguous completeness).

Regardless of the original motivation, one may view the ZAM model as a simple and natural information-theoretic analogue of (non-interactive) zero-knowledge proofs where instead of restricting the computational power of the verifier, we split it between two non-communicating parties (just like AM communication games are derived from the computational-complexity notion of AM protocols). As cryptographers, it is therefore natural to ask:

How does the ZAM model relate to other more standard models of information-theoretic secure computation?

As we will later see, answering this question also allows us to make some (modest) progress in understanding the communication complexity of ZAM protocols.

\subsection{Private Simultaneous Message Protocols}

Another, much older, notion of information-theoretically secure communication game was suggested by Feige, Kilian and Naor [FKN94. As in the previous model, there are three (computationallyunbounded) parties: Alice, Bob and a Referee. Here too, an input $(x, y)$ to a public function $f:\{0,1\}^{n} \times\{0,1\}^{n} \rightarrow\{0,1\}$ is split between Alice and Bob, which, in addition, share a common random string $c$. Alice (resp., Bob) should send to the referee a single message $a$ (resp., $b$ ) such that the transcript $(a, b)$ reveals $f(x, y)$ but nothing else. That is, we require two properties: (Correctness) There exists a decoder algorithm Dec which recovers $f(x, y)$ from $(a, b)$ with high probability; and (Privacy) There exists a simulator Sim which, given the value $f(x, y)$, samples the 
joint distribution of the transcript $(a, b)$ up to some small deviation error. (See Section 4 for formal definitions.)

Following [IK97], we refer to such a protocol as a private simultaneous messages (PSM) protocol. A PSM protocol for $f$ can be alternatively viewed as a special type of randomized encoding of $f$ IK00, AIK06, where the output of $f$ is encoded by the output of a randomized function $F((x, y), c)$ such that $F$ can be written as $F((x, y), c)=\left(F_{1}(x, c), F_{2}(y, c)\right)$. This is referred to as a "2-decomposable" encoding in Ish13]. (See Remark 4.5.)

\subsection{ZAM vs. PSM}

Our goal will be to relate ZAM protocols to PSM protocols. Since the latter object is well studied and strongly "connected" to other information-theoretic notions (cf. [BIKK14]), such a connection will allow us to place the new ZAM in our well-explored world of information-theoretic cryptography.

Observe that ZAM and PSM share some syntactic similarities (illustrated in Figure 1). In both cases, the input is shared between Alice and Bob and the third party holds no input. Furthermore, in both cases the communication pattern consists of a single message. On the other side, in ZAM the third party (Merlin) attempts to convince Alice and Bob that the joint input is mapped to 1, and so the communication goes from Merlin to Alice/Bob who generate the output (accept/reject). In contrast, in a PSM protocol, the messages are sent in the other direction: from Alice and Bob to the third party (the Referee) who ends up with the output. In addition, the privacy guarantee looks somewhat different. For ZAM, privacy is defined with respect to an external observer and only over 1-inputs, whereas soundness is defined with respect to the parties (Alice and Bob) who hold the input $(x, y)$. (Indeed, an external observer cannot even tell whether the joint input $(x, y)$ is a 0-input.) Accordingly, in the ZAM model, correctness and privacy are essentially two different concerns that involve different parties. In contrast, for PSM protocols privacy should hold with respect to the view of the receiver who should still be able to decode.

The picture becomes even more confusing when looking at existing constructions. On one hand, the general ZAM constructions presented by [GPW15, Theorem 6] (which use a reduction to Disjointness) seem more elementary than the simplest PSM protocols of [FKN94]. On the other hand, there are ZAM constructions which share common ingredients with existing PSM protocols. Concretely, the branching-program (BP) representation of the underlying function have been used both in the context of PSM [FKN94, IK97] and in the context of ZAM [GPW15, Theorem 1]. (It should be mentioned that there is a quadratic gap between the complexity of the two constructions.) Finally, both in ZAM and in PSM, it is known that any function $f:\{0,1\}^{n} \times\{0,1\}^{n} \rightarrow\{0,1\}$ admits a protocol with exponential complexity, but the best known lower-bound is only linear in $n$. Overall, it is not clear whether these relations are coincidental or point to a deeper connection between the two models 1

\section{Our Results}

We prove that ZAM protocols and PSM protocols are intimately related. Roughly speaking, we will show that the inverse of ZAM is PSM and vice versa. Therefore, the randomness complexity

\footnotetext{
${ }^{1}$ The authors of GPW15] seem to suggest that there is no a-priori obvious connection between the two models. Indeed, they explicitly mention PSM as "a different model of private two-party computation, [...] where the best upper and lower bounds are also exponential and linear."
} 

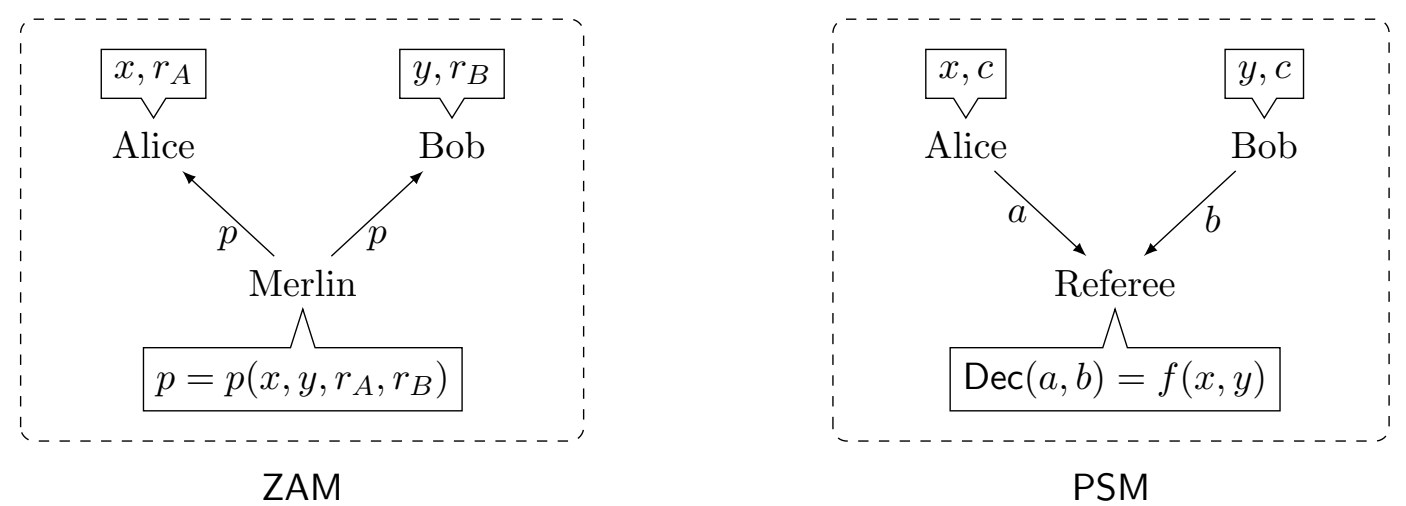

Figure 1: Flow of messages

of ZAM essentially corresponds to the communication complexity of PSM and the communication complexity of ZAM essentially corresponds to the randomness complexity of PSM. This relation works in both directions where different variants of PSM are being used. We exploit this relation to obtain (modest) improvements in the complexity of ZAM and the complexity of some variants of PSM (e.g., Conditional Disclosure of Secrets). We proceed with a formal statement of our results. See Figure 2 for an overview of our transformations.

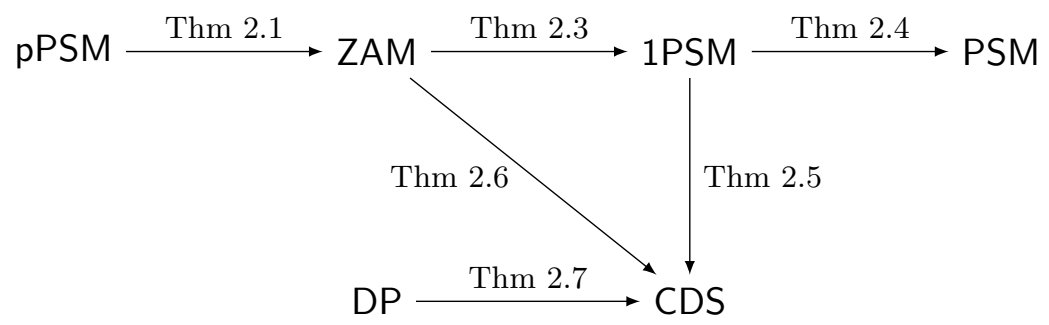

Figure 2: Overview of the constructions

\subsection{From Perfect PSM to ZAM}

We begin by showing that a special form of perfect PSM protocols (referred to pPSM) yields ZAM protocols.

Theorem 2.1. Let $f$ be a function with a pPSM protocol that has communication complexity $t$ and randomness complexity $s$. Then $f$ has a $1 / 2$-sound ZAM scheme with randomness complexity of $t$ and communication complexity of $s+1$.

A pPSM protocol is a PSM in which both correctness and privacy are required to be errorless (perfect), and, in addition, the encoding should satisfy some regularity properties ${ }^{2}$

\footnotetext{
${ }^{2}$ Essentially, the range of $F=\left(F_{1}, F_{2}\right)$ can be partitioned into two equal sets $S_{0}$ and $S_{1}$ and for every input $(x, y)$ the function $F_{x, y}(c)$ that maps the randomness $c$ to the transcript $(a, b)$ forms a bijection from the randomness space to the set $S_{f(x)}$. In the context of randomized encoding, this notion was originally referred to as perfect randomized encoding [AIK06]. See Section 4 for formal definitions.
} 
To prove the theorem, we use the combinatorial properties of the perfect encoding to define a new function $g(x, y, p)=\left(g_{1}(x, p), g_{2}(y, p)\right)$ which, when restricted to a 1 -input $(x, y)$, forms a bijection from the randomness space to the output space, and when $(x, y)$ is a 0 -input the restricted function $g(x, y, \cdot)$ covers only half of the range. Given such a function, it is not hard to design a ZAM: Alice (resp., Bob) samples a random point $r_{A}$ in the range of $g_{1}$ (resp., $r_{B}$ in the range of $g_{2}$ ), and accepts a proof $p$ if $p$ is a preimage of $r_{A}$ under $g_{1}$ (resp. $p$ is a preimage of $r_{B}$ under $g_{2}$ ). It is not hard to verify that the protocol satisfies Unambiguous completeness, $1 / 2$-soundness and zero-information. (See Section 5.)

Although the notion of pPSM looks strong, we note that all known general PSM protocols are perfect. (See Appendices A and B, By plugging in the best known protocol from [BIKK14], we derive the following corollary.

Corollary 2.2. Every function $f:\{0,1\}^{n} \times\{0,1\}^{n} \rightarrow\{0,1\}$ has a ZAM with communication complexity and randomness complexity of $O\left(2^{n / 2}\right)$.

Previously, the best known upper-bound for the ZAM complexity of a general function $f$ was $O\left(2^{n}\right)$ [GPW15]. Using known constructions of BP-based pPSM, we can also re-prove the fact that ZAM complexity is at most polynomial in the size of the BP that computes $f$. (Though, our polynomial is worse than the one achieved by GPW15.)

\subsection{From ZAM to One-Sided PSM}

We move on to study the converse relation. Namely, whether ZAM can be used to derive PSM. For this, we consider a relexation of PSM in which privacy should hold only with respect to 1inputs. In the randomized encoding literature, this notion is referred to as semi-private randomized encoding [AIK06, AIK15]. In the context of PSM protocols we refer to this variant as 1PSM.

Theorem 2.3. Let $f:\{0,1\}^{n} \times\{0,1\}^{n} \rightarrow\{0,1\}$ be a function with a $\delta$-sound ZAM protocol that has communication complexity $\ell$ and randomness complexity $m$. Then, for all $k \in \mathbb{N}$, the following hold:

1. $f$ has $\left(2^{2 n} \delta^{k}\right)$-correct and 0-private 1PSM with communication complexity of $k m$ and $2 \mathrm{~km}$ bits of shared randomness.

2. $f$ has $\left(2^{2 n} \delta^{k}+2^{-\ell k}\right)$-correct and $\left(2^{-\ell k}\right)$-private $1 \mathrm{PSM}$ with communication complexity of $k m$ and $2 \ell k$ bits of shared randomness.

In particular, if the underlying ZAM protocol has a constant error (e.g., $\delta=1 / 2$ ), we can get a 1PSM with an exponential small error of $\exp (-\Omega(n))$ at the expense of a linear overhead in the complexity, i.e., communication complexity and randomness complexity of $O(n m)$ and $O(\ell n)$, respectively.

Both parts of the theorem are proven by "inverting" the ZAM scheme. That is, as a common randomness Alice and Bob will take a proof $p$ sampled according to the ZAM's accepting distribution. Since each proof forms a rectangle, Alice and Bob can locally sample a random point $\left(r_{A}, r_{B}\right)$ from $p$ 's rectangle (Alice samples $r_{A}$ and Bob samples $r_{B}$ ). The 1PSM's encoding functions output the sampled point $\left(r_{A}, r_{B}\right)$. We show that if $(x, y)$ is a 1-input then $\left(r_{A}, r_{B}\right)$ is distributed uniformly, while in the case of the 0 -input the sampled point belongs to some specific set $Z$ that covers only 
a small fraction of the point space. Therefore, the 1PSM's decoder outputs 0 if the sampled point is in $Z$ and 1 , otherwise.

The difference between the two parts of Theorem 2.3 lies in the way that the common randomness is sampled. In the first part we sample $p$ according to the exact ZAM's accepting distribution, whereas in the second part we compromise on imperfect sampling. This allows us to reduce the length of the shared randomness in 1PSM at the expense of introducing the sampling error in privacy and correctness. The proof of the theorem appears in Section 6 .

\subsection{From 1PSM to PSM}

Theorem 2.3 shows that a ZAM protocol with low randomness complexity implies communicationefficient 1PSM protocol. However, the latter object is not well-studied and one may suspect that, for one-sided privacy, such low-communication 1PSM protocols may be easily achievable. The following theorem shows that this is unlikely by relating the worst-case communication complexity of 1PSM to the worst-case communication complexity of general PSM (here "worst case" ranges over all functions of given input length.)

Theorem 2.4. Assume that for all $n$, each function $f:\{0,1\}^{n} \times\{0,1\}^{n} \rightarrow\{0,1\}$ has a $\delta(n)$ correct $\varepsilon(n)$-private 1PSM protocol with communication complexity $t(n)$ and randomness complexity $s(n)$. Then, each $f$ has a $[\delta(n)+\delta(t(n))]$-correct $\max (\varepsilon(n), \delta(n)+\varepsilon(t(n)))$-private PSM protocol with communication complexity $t(t(n))$ and randomness complexity $s(n)+s(t(n))$. In particular, if every such $f$ has a 1PSM with poly $(n)$ communication and randomness, and negligible privacy and correctness errors of $n^{-\omega(1)}$, then every $f$ has a PSM with poly $(n)$ communication and randomness, and negligible privacy and correctness errors of $n^{-\omega(1)}$.

An important open question in information-theoretic cryptography is whether every function $f:\{0,1\}^{n} \times\{0,1\}^{n} \rightarrow\{0,1\}$ admits a PSM whose communication and randomness complexity are polynomial in $n$ and its privacy and correctness errors are negligible in $n$. Therefore, by Theorem 2.4 constructing 1PSM with such parameters would be considered to be a major breakthrough. Together with Theorem 2.3 , we conclude that it will be highly non-trivial to discover randomness-efficient ZAM protocols for general functions.

\subsection{Constructing CDS}

In the CDS model [GIKM00, Alice holds an input $x$ and Bob holds an input $y$, and, in addition, both parties hold a common secret bit $b$. The referee, Carol, holds both $x$ and $y$, but it does not know the secret $b$. Similarly to the PSM case, Alice and Bob use shared randomness to compute the messages $m_{1}$ and $m_{2}$ that are sent to Carol. The CDS requires that Carol can recover $b$ from $\left(m_{1}, m_{2}\right)$ iff $f(x, y)=1$. Moving to the complement $\bar{f}=1-f$ of $f$, one can view the CDS model as a variant of 1PSM, in which the privacy leakage in case of 0 -inputs is full, i.e., given the messages sent by Alice and Bob, one can recover their secret $b$ but on 1-input $b$ remains secret. (Note that $x$ and $y$ are assumed to be public in both cases.) Indeed, it is not hard to prove the following observation.

Theorem 2.5. Assume that the function $f$ has a $\delta$-complete $\varepsilon$-private $1 \mathrm{PSM}$ with communication complexity $t$ and randomness complexity s. Then the function $\bar{f}=1-f$ has a $\delta$-correct and $\varepsilon$-private CDS scheme with communication complexity $t$ and randomness complexity $s$. 
Clearly, one can combine the above theorem with the ZAM to 1PSM transformation and get a transformation from ZAM to CDS. However, one can do better by using a direct construction that avoids the overhead in the ZAM to 1PSM transformation of Theorem 2.3.

Theorem 2.6. Assume that the function $f:\{0,1\}^{n} \times\{0,1\}^{n} \rightarrow\{0,1\}$ has a $\delta$-sound ZAM protocol with communication complexity $\ell$ and randomness complexity $m$. Then the following hold.

1. The function $\bar{f}=1-f$ has a $\delta$-correct and 0 -private CDS with communication complexity $m$ and randomness complexity $2 m$.

2. For any $t \in \mathbb{N}$, the function $\bar{f}$ has a $\left(\delta+2^{-t}\right)$-correct and $\left(2^{-t}\right)$-private $\operatorname{CDS}$ with communication complexity $m$ and randomness complexity $(\ell+t)$.

The communication complexity of CDS protocols was studied in several previous works. Recently, it was shown by Ishai and Wee [W14] that the CDS complexity of $f$ is linear in the size of the arithmetic branching program (ABP). (This improves the previous quadratic upper-bound of [GIKM00].) We can reprove this result by combining Theorem 2.6 with the ZAM construction of [GPW15] whose complexity is also linear in the ABP size of $f$. Interestingly, the resulting CDS protocol is different from the construction of Ishai and Wee [IW14], and can be extended to work with dependency programs (DP). The latter model was introduced in [PS98] and can be viewed as a generalization of arithmetic branching program. (See Section 8 for a formal definition.) By applying the ideas of [GPW15], we derive the following result.

Theorem 2.7. Assume that the function $f$ has a dependency program of size $m$. Then, for every $t \in \mathbb{N}$, the function $f$ has an $2^{-t}$-correct perfectly private CDS scheme with randomness complexity and communication complexity of $O(m \cdot t)$.

The theorem extends to the case where the secret is a field element (see Theorem 8.2) and to the case where $f$ is computed by an arithmetic dependency program and so its inputs are also field elements (see Remark 8.4). To the best of our knowledge, Theorem 2.7 yields the first CDS whose complexity is linear in the dependency program of the underlying function. This is incomparable to the best previous result, implicit in [IW14, Section 7], which achieves linear dependency in the size of the arithmetic span program (ASP) [KW93] that computes $f{ }^{3}$ Indeed, it is known that the size of the smallest dependency program of a function is polynomially related to the size of its smallest span program, but the transformation from one model to the other may incur some polynomial overhead [BG99]. Hence, for some functions, Theorem 2.7 can potentially lead to polynomial improvement over the ASP (and ABP) based schemes. On the other hand, the construction of [IW14] achieves perfect correctness, while our construction suffers from a non-zero decoding error ${ }^{4}$ We further mention that our construction can be viewed as dual to the construction of [IW14]; See Remark 8.5. Finally, we note that CDS protocols have recently found applications in Attribute-Based Encryption (see [GKW15]). For this application, the CDS is required to satisfy some linearity properties which hold for our CDS-based construction. (See Remark 8.3.)

\footnotetext{
${ }^{3}$ Arithmetic span programs [KW93] can emulate ABPs with constant overhead, while the converse is known only with polynomial overhead BG99. Hence, the ASP-based CDS subsume, in terms of complexity, the abovementioned ABP-based construction.

${ }^{4}$ Moreover, the ABP-based construction of IW14 applies not only to CDS, but also to a more general notion of partial garbling schemes which can be viewed as an intermediate notion between CDS and PSM.
} 


\section{Preliminaries}

For an integer $n \in \mathbb{N}$, let $[n]=\{1, \ldots, n\}$. The complement of a bit $b$ is denoted by $\bar{b}=1-b$. For a set $S$, we let $S^{k}$ be the set of all possible $k$-tuples with entries in $S$, and for a distribution $D$, we let $D^{k}$ be the probability distribution over $k$-tuples such that each tuple's element is drawn according to $D$. We let $s \stackrel{R}{\leftarrow} S$ denote an element that is sampled uniformly at random from the finite set $S$. The uniform distribution over $n$-bit strings is denoted by $U_{n}$. For a boolean function $f: S \rightarrow\{0,1\}$, we say that $x \in S$ is 0 -input if $f(x)=0$, and is 1-input if $f(x)=1$. A subset $R$ of a product set $A \times B$ is a rectangle if $R=A^{\prime} \times B^{\prime}$ for some $A^{\prime} \subseteq X$ and $B^{\prime} \subseteq Y$.

The statistical distance between two random variables, $X$ and $Y$, denoted by $\Delta(X ; Y)$ is defined by $\Delta(X ; Y):=\frac{1}{2} \sum_{z}|\operatorname{Pr}[X=z]-\operatorname{Pr}[Y=z]|$. We will also use statistical distance for probability distributions, where for a probability distribution $D$ the value $\operatorname{Pr}[D=z]$ is defined to be $D(z)$.

We write $\Delta_{x_{1} \stackrel{R}{\leftarrow} D_{1}, \ldots, x_{k} \stackrel{R}{\leftarrow} D_{k}}\left(F\left(x_{1}, \ldots, x_{k}\right) ; G\left(x_{1}, \ldots, x_{k}\right)\right)$ to denote the statistical distance between two distributions obtained as a result of sampling $x_{i}$ 's from $D_{i}$ 's and applying the functions $F$ and $G$ to $\left(x_{1}, \ldots, x_{k}\right)$, respectively. We use the following facts about the statistical distance. For every distributions $X$ and $Y$ and a function $F$ (possibly randomized), we have that $\Delta(F(X), F(Y)) \leq \Delta(X, Y)$. In particular, for a boolean function $F$ this implies that $\operatorname{Pr}[F(X)=$ $1] \leq \operatorname{Pr}[F(Y)=1]+\Delta(X ; Y)$.

For a sequence of probability distributions $\left(D_{1}, \ldots, D_{k}\right)$ and a probability vector $W=\left(w_{1}, \ldots, w_{k}\right)$ we let $Z=\sum w_{i} D_{i}$ denote the "mixture distribution" obtained by sampling an index $i \in[k]$ according to $W$ and then outputting an element $z \stackrel{R}{\leftarrow} D_{i}$.

Lemma 3.1. For any distribution $Z=\sum w_{i} D_{i}$ and probability distribution $S$, it holds that

$$
\Delta(S ; Z) \leq \sum_{i=1}^{k} w_{i} \Delta\left(S ; D_{i}\right)
$$

Proof. By the definition of statistical distance we can write $\Delta(S ; Z)$ as

$$
\begin{aligned}
\frac{1}{2} \sum_{z}\left|S(z)-\sum_{i=1}^{k} w_{i} D_{i}(z)\right| & =\frac{1}{2} \sum_{z}\left|\sum_{i=1}^{k} w_{i}\left(S(z)-D_{i}(z)\right)\right| \\
& \leq \frac{1}{2} \sum_{z} \sum_{i=1}^{k} w_{i}\left|S(z)-D_{i}(z)\right| \\
& =\frac{1}{2} \sum_{i=1}^{k} w_{i} \sum_{z}\left|S(z)-D_{i}(z)\right| \\
& =\sum_{i=1}^{k} w_{i} \Delta\left(S ; D_{i}\right) .
\end{aligned}
$$




\section{Definitions}

\subsection{PSM-Based Models}

Definition 4.1 (PSM). Let $f:\{0,1\}^{n} \times\{0,1\}^{n} \rightarrow\{0,1\}$ be a boolean function. We say that a pair of (possibly randomized $\left(^{5}\right.$ ) encoding algorithms $F_{1}, F_{2}:\{0,1\}^{n} \times\{0,1\}^{s} \rightarrow\{0,1\}^{t}$ are PSM for $f$ if the function $F(x, y, c)=\left(F_{1}(x, c), F_{2}(y, c)\right)$ that corresponds to the joint computation of $F_{1}$ and $F_{2}$ on a common $c$, satisfy the following properties:

$\delta$-CoRRectness: There exists a deterministic algorithm Dec, called decoder, such that for every input $(x, y)$ we have that

$$
\operatorname{Pr}_{c \stackrel{R}{\leftarrow}\{0,1\}^{s}}[\operatorname{Dec}(F(x, y, c)) \neq f(x, y)] \leq \delta .
$$

E-PRIVACY: There exists a randomized algorithm (simulator) Sim such that for any input $(x, y)$ it holds that

$$
\underset{c \stackrel{R}{\leftarrow}\{0,1\}^{s}}{\Delta}(\operatorname{Sim}(f(x, y)) ; F(x, y, c)) \leq \varepsilon .
$$

The communication complexity of the PSM protocol is defined as the encoding length $t$, and the randomness complexity of the protocol is defined as the length $s$ of the common randomness.

One can also consider relaxations of this definition that are private only on a subset of inputs. We study such a relaxation 1PSM [AIK06, AIK15] that is required to be private only on 1-inputs: $\varepsilon$-PRIVACY ON 1-INPUTS: There exists a simulator Sim such that for any 1-input $(x, y)$ of $f$ it holds that

$$
\underset{c \leftarrow\{0,1\}^{s}}{\Delta}\left(\operatorname{Sim},\left(F_{1}(x, c), F_{2}(y, c)\right)\right) \leq \varepsilon .
$$

A stronger variant of PSM is captured by the notion of perfect PSM [AIK06].

Definition 4.2 (pPSM). Let $f:\{0,1\}^{n} \times\{0,1\}^{n} \rightarrow\{0,1\}$. A pair of deterministic algorithms $F_{1}, F_{2}:\{0,1\}^{n} \times\{0,1\}^{s} \rightarrow\{0,1\}^{t}$ is a pPSM of $f$ if $\left(F_{1}, F_{2}\right)$ is a 0 -correct, 0-private PSM of $f$ such that:

BALANCE: There exists a 0-private (perfectly private) simulator $\operatorname{Sim}$ such that $\operatorname{Sim}\left(U_{1}\right) \equiv U_{2 t}$.

Stretch-Preservation: We have that $1+s=2 t$.

Remark 4.3 (pPSM - combinatorial view). One can also formulate the pPSM definition combinatorially [AIK06]: For $f$ 's b-input $(x, y)$, let $F_{x y}(c)$ denote the joint output of the encoding $\left(F_{1}(x, c), F_{2}(y, c)\right)$. Let $S_{b}:=\left\{F_{x y}(c) \mid c \in\{0,1\}^{s},(x, y) \in f^{-1}(b)\right\}$ and let $R=\{0,1\}^{t} \times\{0,1\}^{t}$ denote the joint range of $\left(F_{1}, F_{2}\right)$. Then, $\left(F_{1}, F_{2}\right)$ is a pPSM of $f$ if and only if (1) The 0-image $S_{0}$ and the 1-image $S_{1}$ are disjoint; (2) The union of $S_{0}$ and $S_{1}$ equals to the range $R$; and (3) for all $(x, y)$ the function $F_{x y}$ is a bijection on $S_{f(x, y)}$. One can also consider a case when $F_{1}$ and $F_{2}$ have arbitrary ranges, i.e., $F_{i}:\{0,1\}^{n} \times\{0,1\}^{s} \rightarrow\{0,1\}^{t_{i}}$. In this case we say that $\left(F_{1}, F_{2}\right)$ is a pPSM of $f$ if the above conditions hold with respect to the joint range $R=\{0,1\}^{t_{1}} \times\{0,1\}^{t_{2}}$.

\footnotetext{
${ }^{5}$ In the original paper [FKN94, the functions $F_{1}, F_{2}$ are deterministic. We extend this model by allowing Alice and Bob to use local randomness that is assumed to be available freely.
} 
We consider a variant of CDS called conditional disclosure of the common secret [GIKM00]. As in PSM, Alice and Bob hold the inputs $x$ and $y$, respectively, and, in addition, both parties get a secret $b \in\{0,1\}$. The goal is to reveal the secret to an external referee Carol only if some predicate $f(x, y)$ evaluates to 1 . Unlike the PSM model, we assume that Carol knows both $x$ and $y$. Formally, a CDS scheme is defined below.

Definition 4.4 (CDS). Let $f:\{0,1\}^{n} \times\{0,1\}^{n} \rightarrow\{0,1\}$ be a predicate. Let $F_{1}, F_{2}:\{0,1\}^{n} \times$ $\{0,1\} \times\{0,1\}^{s} \rightarrow\{0,1\}^{t}$ be (possibly randomized) encoding algorithms. Then, the pair $\left(F_{1}, F_{2}\right)$ is a CDS scheme for $f$ if and only if the function $F(x, y, b, c)=\left(F_{1}(x, b, c), F_{2}(y, b, c)\right)$ that corresponds to the joint computation of $F_{1}$ and $F_{2}$ on a common $b$ and $c$, satisfies the following properties:

$\delta$-Correctness: There exists a deterministic algorithm Dec, called a decoder, such that for every 1-input $(x, y)$ of $f$ and any secret $b \in\{0,1\}$ we have that

$$
\operatorname{Pr}_{c \leftarrow\{0,1\}^{s}}[\operatorname{Dec}(x, y, F(x, y, b, c)) \neq b] \leq \delta .
$$

$\varepsilon$-PRIVACY: There exists a simulator Sim such that for every 0-input $(x, y)$ of $f$ and any secret $b \in\{0,1\}$ it holds that

$$
\underset{c \stackrel{R}{\leftarrow}\{0,1\}^{s}}{\Delta}(\operatorname{Sim}(x, y) ; F(x, y, b, c)) \leq \varepsilon .
$$

Similarly to PSM, the communication complexity of the CDS protocol is $t$ and its randomness complexity is $s$.

The above definition naturally extends to the case where the secret comes from some non-binary domain $B$, and where the domain of the randomness and of the output of $F_{1}$ and $F_{2}$ is taken to be some arbitrary finite set. (When the output domain $Z_{1}$ of $F_{1}$ and $Z_{2}$ of $F_{2}$ differ, we define the communication complexity to be $\max _{i} \log \left|Z_{i}\right|$.)

Remark 4.5 (CDS and PSM as Randomized Encoding). We can view PSM and CDS protocols under the framework of randomized encodings of functions (RE) [IK00, AIK06]. Formally, a function $F(x, y, c)$ is a $\delta$-correct $\varepsilon$-private $R E$ of $f(x, y)$ if $F(x, y)$ satisfies Eq. (1) and Eq. (2) from Definition 4.1. Under this terminology, PSM is simply an encoding $F(x, y, c)$ which can be decomposed into two parts, $F_{1}$ which depends on $x$ and $c$ but not on $y$ and $F_{2}$ which depends on $y$ and $c$ but not on $x$. Similarly, the notion of PPSM and 1PSM can be derived by considering 2-decomposable perfect encodings and 2-decomposable encoding with 1-sided privacy. We further mention that a CDS can be also viewed as a randomized encoding. Indeed, $\left(F_{1}, F_{2}\right)$ is a CDS of $f$ if and only if $F(x, y, b, c)=\left(x, y, F_{1}(x, b, c), F_{2}(y, b, c)\right)$ encodes the (non-boolean) function $g(x, y, b)=(x, y, f(x, y) \wedge b)$.

\subsection{ZAM}

Definition 4.6 (ZAM). Let $f:\{0,1\}^{n} \times\{0,1\}^{n} \rightarrow\{0,1\}$. We say that a pair of deterministic boolean functions $A, B:\{0,1\}^{n} \times\{0,1\}^{m} \times\{0,1\}^{\ell} \rightarrow\{0,1\}$ is a ZAM for $f$ if the predicate $\operatorname{Accept}\left(x, y, r_{A}, r_{B}, p\right)=A\left(x, r_{A}, p\right) \wedge B\left(y, r_{B}, p\right)$ satisfies the following properties:

Unambiguous Completeness: For any 1-input $(x, y)$ and any randomness $\left(r_{A}, r_{B}\right) \in\{0,1\}^{m} \times$ $\{0,1\}^{m}$ there exists a unique $p \in\{0,1\}^{\ell}$ such that $\operatorname{Accept}\left(x, y, r_{A}, r_{B}, p\right)=1$. 
Zero Information: There exists a distribution $D$ on the proof space $\{0,1\}^{\ell}$ such that for any 1-input $(x, y)$ we have that

$$
\forall p \in\{0,1\}^{\ell} D(p)=\underset{r_{A}, r_{B} \stackrel{R}{\leftarrow}\{0,1\}^{m}}{\operatorname{Pr}}\left[\operatorname{Accept}\left(x, y, r_{A}, r_{B}, p\right)=1\right] .
$$

The distribution $D$ is called the accepting distribution.

$\delta$-SOUnDNESS: For any 0-input $(x, y)$ it holds that

$$
\underset{r_{A}, r_{B} \stackrel{R}{R}\{0,1\}^{m}}{\operatorname{Pr}}\left[\exists p \in\{0,1\}^{\ell}: \operatorname{Accept}\left(x, y, r_{A}, r_{B}, p\right)=1\right] \leq \delta .
$$

The communication complexity (resp., randomness complexity) of the ZAM protocol is defined as the length $\ell$ of the proof (resp., the length $m$ of the local randomness).

The Zero Information property asserts that for every accepting input $(x, y)$ the distribution $D_{x, y}$, obtained by sampling $r_{A}$ and $r_{B}$ and outputting the (unique) proof $p$ which is accepted by Alice and Bob, is identical to a single universal distribution $D$.

Following [GPW15, we sometimes refer to the proofs as "rectangles" because for each $(x, y)$ a proof $p$ naturally corresponds to a set of points

$$
\left\{\left(r_{A}, r_{B}\right): \operatorname{Accept}\left(x, y, r_{A}, r_{B}, p\right)=1\right\}=\left\{r_{A}: A\left(x, r_{A}, p\right)=1\right\} \times\left\{r_{B}: B\left(y, r_{B}, p\right)=1\right\}
$$

which forms a rectangle in $\{0,1\}^{m} \times\{0,1\}^{m}$.

\section{From pPSM to ZAM}

In this section we construct a ZAM scheme from a pPSM protocol. By exploiting the combinatorial structure of pPSM, for each input $(x, y)$ we construct a function $h_{x y}$ that is a bijection if $(x, y)$ is a 1-input and is two-to-one if $(x, y)$ is a 0-input. In the constructed ZAM scheme Alice and Bob use their local randomness to sample a uniform point in $h$ 's range (Alice samples its $x$-coordinate $r_{A}$ and Bob samples its $y$-coordinate $\left.r_{B}\right)$. Merlin's proof is the preimage $p$ for the sampled point, i.e., a point $p$ such that $h_{x y}(p)=\left(r_{A}, r_{B}\right)$. In order to accept the proof $p$, Alice and Bob verify that it is a preimage for the sampled point $\left(r_{A}, r_{B}\right)$.

First, the constructed ZAM is unambiguously complete because $h_{x y}$ is a bijection if $(x, y)$ is a 1-input of $f$. Second, the constructed ZAM satisfies the zero-information property because the distribution of the accepted proofs is uniform. Third, the constructed ZAM is sound, because if $(x, y)$ is a 0 -input, then $h_{x y}$ is two-to-one, implying that with probability at least $1 / 2$ no preimage exists.

Theorem 2.1. Let $f$ be a function with a pPSM protocol that has communication complexity $t$ and randomness complexity $s$. Then $f$ has a $1 / 2$-sound ZAM scheme with randomness complexity of $t$ and communication complexity of $s+1$.

Proof. Let $f:\{0,1\}^{n} \times\{0,1\}^{n} \rightarrow\{0,1\}$ be a function with a pPSM $F_{1}, F_{2}:\{0,1\}^{n} \times\{0,1\}^{s} \rightarrow$ $\{0,1\}^{t}$. We show that there exists a $1 / 2$-sound ZAM protocol for $f$ with Alice's and Bob's local randomness spaces $\{0,1\}^{m}$ and proof space $\{0,1\}^{\ell}$ where $m=t$ and $\ell=2 t$. 
First, we prove some auxiliary statement about pPSM. Let $g(x, y, c):=\left(F_{1}(x, c), F_{2}(y, c)\right)$. For any $(x, y)$, we define a new function $h_{x y}:\{0,1\}^{s} \times\{0,1\} \rightarrow\{0,1\}^{t} \times\{0,1\}^{t}$ as follows.

$$
h_{x y}(c, b):=\left\{\begin{array}{l}
g(x, y, c), \text { if } b=0 ; \\
g\left(x_{0}, y_{0}, c\right), \text { if } b=1\left(\text { where }\left(x_{0}, y_{0}\right) \text { is a canonical 0-input for } f\right) .
\end{array}\right.
$$

The function $h$ satisfies the following useful properties as follows from the combinatorial view of pPSM (Remark 4.3).

Fact 5.1. If $(x, y)$ is a 1-input for $f$, then the function $h_{x y}$ is a bijection. Otherwise, if $(x, y)$ is a 0-input for $f$, then the image of the function $h_{x y}$ covers exactly half of the range $\{0,1\}^{t} \times\{0,1\}^{t}$.

We now describe a ZAM protocol for $f$ in which the local randomness of Alice and Bob is sampled from $\{0,1\}^{t}$, and the proof space is $\{0,1\}^{s} \times\{0,1\}$. Recall that $\left(F_{1}, F_{2}\right)$ is a pPSM and therefore $s+1=2 t$ and $\{0,1\}^{s} \times\{0,1\}=\{0,1\}^{2 t}$. The ZAM's accepting functions $A, B$ are defined as follows:

$$
\begin{aligned}
& A\left(x, m_{1},(c, b)\right)=\left\{\begin{array}{l}
1, \text { if }\left(m_{1}=F_{1}(x, c) \text { and } b=0\right) \text { or }\left(m_{1}=F_{1}\left(x_{0}, c\right) \text { and } b=1\right) ; \\
0, \text { otherwise. }
\end{array}\right. \\
& B\left(y, m_{2},(c, b)\right)=\left\{\begin{array}{l}
1, \text { if }\left(m_{2}=F_{2}(y, c) \text { and } b=0\right) \text { or }\left(m_{2}=F_{2}\left(y_{0}, c\right) \text { and } b=1\right) ; \\
0, \text { otherwise. }
\end{array}\right.
\end{aligned}
$$

Observe that the following equivalence holds.

Claim 5.2. $\forall x, y, c, b, m_{1}, m_{2}\left[h_{x y}(c, b)=\left(m_{1}, m_{2}\right)\right] \Leftrightarrow\left[A\left(x, m_{1},(c, b)\right)=1=B\left(y, m_{2},(c, b)\right)\right]$.

Now we verify that $(A, B)$ is ZAM for $f$ :

Unambiguous Completeness: Consider any $f$ 's 1 -input $(x, y)$ and take any $\left(m_{1}, m_{2}\right) \in\{0,1\}^{t} \times$ $\{0,1\}^{t}$. Since $(x, y)$ is a 1 -input for $f$, we have that $h_{x y}$ is a bijection. This means that there exists a unique $(c, b)$ such that $h_{x y}(c, b)=\left(m_{1}, m_{2}\right)$. By Claim 5.2, this proof $(c, b)$ is the only proof which is accepted by both Alice and Bob when the randomness is set to $m_{1}, m_{2}$.

Zero Information: We show that the accepting distribution is uniform, i.e., for any 1-input $(x, y)$ and for any $p \in\{0,1\}^{s} \times\{0,1\}$ it holds that

$$
\underset{r_{A}, r_{B} \stackrel{R}{\leftarrow}\{0,1\}^{t}}{\operatorname{Pr}}\left[A\left(x, r_{A}, p\right)=1=B\left(y, r_{B}, p\right)\right]=2^{-2 t} .
$$

Take any 1 -input $(x, y)$. Since $(x, y)$ is a 1 -input for $f$, we have that $h_{x y}$ is a bijection. Hence, there exists a unique $\left(m_{1}^{*}, m_{2}^{*}\right) \in\{0,1\}^{n} \times\{0,1\}^{n}$ such that $h_{x y}(c, b)=\left(m_{1}^{*}, m_{2}^{*}\right)$. By Claim 5.2 this means that Alice and Bob accept only this $\left(m_{1}^{*}, m_{2}^{*}\right)$. Hence, for all proofs $p$ we have that

$$
\underset{r_{A}, r_{B}{ }^{R}\{0,1\}^{t}}{\operatorname{Pr}}\left[A\left(x, r_{A}, p\right)=1=B\left(y, r_{B}, p\right)\right]=\underset{r_{A}, r_{B}{ }^{R}\{0,1\}^{t}}{\operatorname{Pr}}\left[r_{A}=m_{1}^{*}, r_{B}=m_{2}^{*}\right]=2^{-2 t} .
$$

1/2-Soundness: Fix some 0 -input $(x, y)$, and recall that the image $H$ of $h_{x y}$ covers exactly half of the range $\{0,1\}^{t} \times\{0,1\}^{t}$, i.e., $|H|=\left|\{0,1\}^{t} \times\{0,1\}^{t}\right| / 2$. It follows that, with probability $1 / 2$, the randomness of Alice and $\mathrm{Bob}\left(m_{1}, m_{2}\right)$ chosen randomly from $\{0,1\}^{t} \times\{0,1\}^{t}$ lands outside $H$. In this case, the set $h_{x y}^{-1}\left(m_{1}, m_{2}\right)$ is empty and so there is no proof $(c, b)$ that will be accepted. 


\section{From ZAM to 1PSM}

In this section we construct 1PSM protocols from a ZAM scheme and prove Theorem 2.3 (restated here for convenience).

Theorem 2.3. Let $f:\{0,1\}^{n} \times\{0,1\}^{n} \rightarrow\{0,1\}$ be a function with a $\delta$-sound ZAM protocol that has communication complexity $\ell$ and randomness complexity $m$. Then, for all $k \in \mathbb{N}$, the following hold:

1. $f$ has $\left(2^{2 n} \delta^{k}\right)$-correct and 0-private 1PSM with communication complexity of $\mathrm{km}$ and $2 \mathrm{~km}$ bits of shared randomness.

2. $f$ has $\left(2^{2 n} \delta^{k}+2^{-\ell k}\right)$-correct and $\left(2^{-\ell k}\right)$-private 1PSM with communication complexity of $k m$ and $2 \ell k$ bits of shared randomness.

Proof. Let $f:\{0,1\}^{n} \times\{0,1\}^{n} \rightarrow\{0,1\}$ be a function with a $\delta$-sound ZAM protocol $(A, B)$ with Alice's and Bob's local randomness spaces $\{0,1\}^{m}$ and the proof space $\{0,1\}^{\ell}$. Fix some integer $k$. We start by constructing the first 1PSM protocol.

We first define some additional notation and prove auxiliary claims. For a pair of inputs $(x, y)$ let

$$
E_{x y}:=\left\{\left(r_{A}, r_{B}\right) \in\{0,1\}^{m} \times\{0,1\}^{m} \mid \exists p: A\left(x, r_{A}, p\right)=1=B\left(y, r_{B}, p\right)\right\}
$$

and $Z:=\bigcup_{(x, y) \in f^{-1}(0)} E_{x y}^{k}$.

Claim 6.1. $|Z| \leq 2^{2 n}\left(\delta 2^{2 m}\right)^{k}$.

Proof. By the soundness property of ZAM, we have that $\left|E_{x y}\right| \leq \delta 2^{2 m}$ for any 0 -input $(x, y)$. Hence, each $\left|E_{x y}^{k}\right| \leq\left(\delta 2^{2 m}\right)^{k}$. We conclude that

$$
|Z|=\left|\bigcup_{(x, y) \in f^{-1}(0)} E_{x y}^{k}\right| \leq \sum_{(x, y) \in f^{-1}(0)}\left|E_{x y}^{k}\right| \leq 2^{2 n}\left(\delta 2^{2 m}\right)^{k}=\delta^{k} 2^{2 n+2 m k} .
$$

Let $\mathcal{A}_{p}^{x}:=\left\{r_{A} \in\{0,1\}^{m} \mid A\left(x, r_{A}, p\right)=1\right\}$ and $\mathcal{B}_{p}^{y}:=\left\{r_{B} \in\{0,1\}^{m} \mid B\left(y, r_{B}, p\right)=1\right\}$.

Claim 6.2. Let $D_{\mathrm{ACC}}$ be the accepting distribution of ZAM. Then, for any 1-input $(x, y)$ and $p \in\{0,1\}^{\ell}$ we have that $D_{\mathrm{ACC}}(p)=2^{-2 m}\left|\mathcal{A}_{p}^{x}\right|\left|\mathcal{B}_{p}^{y}\right|$.

Proof. By definition

$$
D_{\mathrm{ACC}}(p)=\frac{\left|\left\{\left(r_{A}, r_{B}\right) \in\{0,1\}^{m} \times\{0,1\}^{m} \mid A\left(x, r_{A}, p\right)=1=B\left(y, r_{B}, p\right)\right\}\right|}{\left|\{0,1\}^{m}\right| \cdot\left|\{0,1\}^{m}\right|} .
$$

In order to derive the claim, note that since every proof forms a "rectangle" (see Section 4.2), we have that

$$
\left\{\left(r_{A}, r_{B}\right) \in\{0,1\}^{m} \times\{0,1\}^{m} \mid A\left(x, r_{A}, p\right)=1=B\left(y, r_{B}, p\right)\right\}=\mathcal{A}_{p}^{x} \times \mathcal{B}_{p}^{y} .
$$


We can now describe the encoding algorithms $G_{1}$ and $G_{2}$ and the decoder Dec. First, $G_{1}$ and $G_{2}$ use the shared randomness to sample a proof $p$ according to the accepting distribution. Then $G_{1}$ and $G_{2}$ sample (private) randomness that can lead to the acceptance of $p$ on their input $(x, y)$, i.e., $G_{1}$ computes $a \stackrel{R}{\leftarrow} \mathcal{A}_{p}^{x}$ and $G_{2}$ computes $b \stackrel{R}{\leftarrow} \mathcal{B}_{p}^{y}$. We have that if $f(x, y)=1$ then $(a, b)$ is distributed uniformly, while if $f(x, y)=0$ then $(a, b)$ is sampled from the set $Z$. The task of the decoder is to verify whether it is likely that a point has been sampled from $Z$ or uniformly. This is achieved by repeating the protocol $k$ times. Below is the formal description of the algorithms $G_{1}, G_{2}$, and decoder.

- (Shared Randomness) The common randomness $c \in\{0,1\}^{k \cdot 2 m}$ is used for sampling $k$ independent samples $\left(p_{1}, \ldots, p_{k}\right)$ from $D_{\mathrm{ACC}}$. (Each such sample can be obtained by sampling $r=\left(r_{A}, r_{B}\right) \stackrel{R}{\leftarrow}\{0,1\}^{2 m}$ and outputting the unique proof $p$ that corresponds to $r$ and to some fixed 1-input $\left(x_{0}, y_{0}\right)$.)

- (Encoders) The encoder $G_{1}(x, c)$ outputs $\left(a_{1}, \ldots, a_{k}\right) \stackrel{R}{\leftarrow} \mathcal{A}_{p_{1}}^{x} \times \cdots \times \mathcal{A}_{p_{k}}^{x}$ and the encoder $G_{2}$ outputs $\left(b_{1}, \ldots, b_{k}\right) \stackrel{R}{\leftarrow} \mathcal{B}_{p_{1}}^{y} \times \cdots \times \mathcal{B}_{p_{k}}^{x}$.

- (Decoder) $\operatorname{Dec}\left(\left(a_{1}, \ldots, a_{k}\right),\left(b_{1}, \ldots, b_{k}\right)\right)$ outputs 0 if $\left(\left(a_{1}, b_{1}\right), \ldots,\left(a_{k}, b_{k}\right)\right) \in Z$; otherwise, it outputs 1 .

Let us verify that the proposed protocol is a 1PSM for $f$.

$\left(2^{2 n} \delta^{k}\right)$-Correctness. Since that the decoder never errs on 0-inputs, it suffices to analyze the probability that some 1 -input $(x, y)$ is incorrectly decoded to 0 . Fix some 1 -input $(x, y)$. Below we will show that the message $\vec{s}=\left(\left(a_{1}, b_{1}\right), \ldots,\left(a_{k}, b_{k}\right)\right)$ generated by the encoders $G_{1}$ and $G_{2}$ is uniformly distributed over the set $\left(\{0,1\}^{m} \times\{0,1\}^{m}\right)^{k}$. Hence, the probability that $\vec{s}$ lands in $Z$ (and decoded incorrectly to 0) is exactly $\frac{|Z|}{\left|\left(\{0,1\}^{m} \times\{0,1\}^{m}\right)^{k}\right|}$, which, by Claim 6.1 is upper-bounded by $2^{2 n} \delta^{k}$.

It is left to show that $\vec{s}$ is uniformly distributed. To see this, consider the marginalization of $\left(a_{i}, b_{i}\right)$ 's probability distribution: For a fixed $\left(r_{A}, r_{B}\right)$ we have that

$$
\operatorname{Pr}\left[\left(a_{i}, b_{i}\right)=\left(r_{A}, r_{B}\right)\right]=\sum_{p \in\{0,1\}^{\ell}} \operatorname{Pr}\left[\left(a_{i}, b_{i}\right)=\left(r_{A}, r_{B}\right) \mid p_{i}=p\right] \operatorname{Pr}\left[p_{i}=p\right] .
$$

Because of the unambiguous completeness property of ZAM, we have that there exists a single $p^{*}$ such that $\left(r_{A}, r_{B}\right) \in \mathcal{A}_{p^{*}}^{x} \times \mathcal{B}_{p^{*}}^{y}$. Hence, all probabilities $\operatorname{Pr}\left[\left(a_{i}, b_{i}\right)=\left(r_{A}, r_{B}\right) \mid p_{i}=p\right]$ are zero, if $p \neq p^{*}$. This implies that

$$
\operatorname{Pr}\left[\left(a_{i}, b_{i}\right)=\left(r_{A}, r_{B}\right)\right]=\operatorname{Pr}\left[\left(a_{i}, b_{i}\right)=\left(r_{A}, r_{B}\right) \mid p_{i}=p^{*}\right] \operatorname{Pr}\left[p_{i}=p^{*}\right] .
$$

We have that $\operatorname{Pr}\left[p_{i}=p\right]=D_{\mathrm{ACC}}(p)=2^{-2 m}\left|\mathcal{A}_{p}^{x}\right|\left|\mathcal{B}_{p}^{y}\right|$ (due to Claim 6.2), and $\operatorname{Pr}\left[\left(a_{i}, b_{i}\right)=\right.$ $\left.\left(r_{A}, r_{B}\right) \mid p_{i}=p^{*}\right]$ is $\frac{1}{\left|\mathcal{A}_{p}^{x}\right| \cdot\left|\mathcal{B}_{p}^{y}\right|}$ by the construction of the encoding functions. Hence, $\operatorname{Pr}\left[\left(a_{i}, b_{i}\right)=\right.$ $\left.\left(r_{A}, r_{B}\right)\right]=2^{-2 m}$. Because all pairs $\left(a_{i}, b_{i}\right)$ are sampled independently, we get that the combined tuple $\vec{s}=\left(\left(a_{1}, b_{1}\right), \ldots,\left(a_{k}, b_{k}\right)\right)$ is sampled uniformly from $\left(\{0,1\}^{m} \times\{0,1\}^{m}\right)^{k}$, as required.

Privacy for 1-inputs. As shown above, if $(x, y)$ is a 1-input, then $\vec{s}$ is uniformly distributed over $\left(\{0,1\}^{m} \times\{0,1\}^{m}\right)^{k}$. Hence, the simulator for proving the privacy property of PSM can be defined as a uniform sampler from $\left(\{0,1\}^{m} \times\{0,1\}^{m}\right)^{k}$. 
The second protocol. The second item of the theorem is proved by using the first protocol, except that the point $\vec{p}=\left(p_{1}, \ldots, p_{k}\right)$ is sampled from a different distribution $D^{\prime}$. For a parameter $t$, the distribution $D^{\prime}$ is simply the distribution $D_{\mathrm{ACC}}^{k}$ discretized into $2^{-(\ell k+t)}$-size intervals. Such $D^{\prime}$ can be sampled using only $\ell k+t$ random bits. Moreover, for each point $\vec{p}$, the difference between $D_{\mathrm{ACC}}^{k}(\vec{p})$ and $D^{\prime}(\vec{p})$ is at most $2^{-(\ell k+t)}$. Since the support of $D_{\mathrm{ACC}}^{k}$ is of size at most $2^{\ell k}$, it follows that $\Delta\left(D^{\prime} ; D_{\mathrm{ACC}}^{k}\right) \leq 2^{-(\ell k+t)} \cdot 2^{\ell k}=2^{-t}$. As a result, we introduce an additional error of $2^{-t}$ in both privacy and correctness. By setting $t$ to $\ell k$, we derive the second 1PSM protocol.

\section{From 1PSM to PSM}

In this section we show how to upgrade a 1PSM protocol into a PSM protocol. We assume that we have a way of constructing 1PSM for all functions. Our main idea is to reduce a construction of a PSM scheme for $f$ to two 1PSM schemes. The first 1PSM scheme computes the function $f$, and

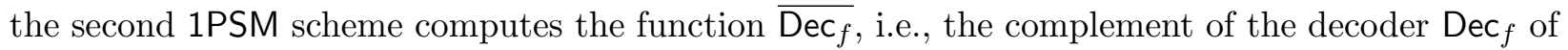
the first scheme. We show how to combine the two schemes such that the first scheme protects the privacy of 1-inputs and the second scheme protects the privacy of 0 -inputs.

Theorem 2.4. Assume that for all $n$, each function $f:\{0,1\}^{n} \times\{0,1\}^{n} \rightarrow\{0,1\}$ has a $\delta(n)$ correct $\varepsilon(n)$-private 1PSM protocol with communication complexity $t(n)$ and randomness complexity $s(n)$. Then, each $f$ has a $[\delta(n)+\delta(t(n))]$-correct $\max (\varepsilon(n), \delta(n)+\varepsilon(t(n)))$-private PSM protocol with communication complexity $t(t(n))$ and randomness complexity $s(n)+s(t(n))$. In particular, if every such $f$ has a 1PSM with poly $(n)$ communication and randomness, and negligible privacy and correctness errors of $n^{-\omega(1)}$, then every $f$ has a PSM with poly $(n)$ communication and randomness, and negligible privacy and correctness errors of $n^{-\omega(1)}$.

Proof. Let $f:\{0,1\}^{n} \times\{0,1\}^{n} \rightarrow\{0,1\}$. Let $F_{1}, F_{2}:\{0,1\}^{n} \times\{0,1\}^{s(n)} \rightarrow\{0,1\}^{t(n)}$ be a $\delta(n)$ correct and $\varepsilon(n)$-private on 1 inputs 1PSM for $f$ with decoder $\operatorname{Dec}_{f}$ and simulator $\operatorname{Sim}_{f}$. Define a function $g:\{0,1\}^{t(n)} \times\{0,1\}^{t(n)} \rightarrow\{0,1\}$ to be $1-\operatorname{Dec}_{f}\left(m_{1}, m_{2}\right)$. Let $G_{1}, G_{2}:\{0,1\}^{t(n)} \times$ $\{0,1\}^{s(t(n))} \rightarrow\{0,1\}^{t(t(n))}$ be a $\delta(t(n))$-correct and $\varepsilon(t(n))$-private on 1 inputs 1PSM for $g$ with decoder $\operatorname{Dec}_{g}$ and simulator $\operatorname{Sim}_{g}$.

We construct a (standard) PSM for $f$ as follows. Let $\{0,1\}^{u}=\{0,1\}^{s(n)} \times\{0,1\}^{s(t(n))}$ be the space of shared randomness, let $\{0,1\}^{v}=\{0,1\}^{t(t(n))}$ be the output space and define the encoding functions $H_{1}, H_{2}:\{0,1\}^{n} \times\{0,1\}^{u} \rightarrow\{0,1\}^{v}$, by

$$
H_{1}(x,(c, r))=G_{1}\left(F_{1}(x, c), r\right) \quad \text { and } \quad H_{2}(y,(c, r))=G_{2}\left(F_{2}(y, c), r\right) .
$$

We show that $\left(H_{1}, H_{2}\right)$ is a PSM by verifying its security properties.

$\delta(n)+\delta(t(n))$-Correctness: On an input $\left(e_{1}, e_{2}\right)$ define the decoding algorithm Dec to output $1-\operatorname{Dec}_{g}\left(e_{1}, e_{2}\right)$. The decoding algorithm Dec works correctly whenever both $\operatorname{Dec}_{g}$ and $\operatorname{Dec}_{f}$ succeed. 
Hence, the error probability for decoding can be bounded as follows:

$$
\begin{aligned}
& \underset{(c, r) \stackrel{\operatorname{Pr}}{\leftarrow}\{0,1\}^{u}}{\ln }\left[\operatorname{Dec}\left(H_{1}(x,(c, r)), H_{2}(y,(c, r))\right) \neq f(x, y)\right] \\
& \left.\left.=\underset{(c, r) \stackrel{R}{\leftarrow}\{0,1\}^{u}}{\operatorname{Pr}}\left[1-\operatorname{Dec}_{g}\left(G_{1}\left(F_{1}(x, c), r\right)\right), G_{2}\left(F_{2}(y, c), r\right)\right)\right) \neq f(x, y)\right] \\
& \leq \underset{c \leftarrow\{0,1\}^{s(n)}}{\operatorname{Pr}}\left[1-\left(1-\left(\operatorname{Dec}_{f}\left(F_{1}(x, c), F_{2}(y, c)\right)\right)\right) \neq f(x, y)\right]+\delta(t(n)) \\
& =\underset{c \leftarrow\{0,1\}^{s(n)}}{\operatorname{Pr}}\left[\operatorname{Dec}_{f}\left(F_{1}(x, c), F_{2}(y, c)\right) \neq f(x, y)\right]+\delta(t(n)) \\
& \leq \delta(n)+\delta(t(n))
\end{aligned}
$$

$\varepsilon$-PRIVACY: We define the simulator Sim as follows: on 0-inputs it outputs $\operatorname{Sim}_{g}$ and on 1-inputs it computes $\operatorname{Sim}_{f}=\left(m_{1}, m_{2}\right)$, randomly samples $r$ from $\{0,1\}^{s(t(n))}$, and outputs $\left(G_{1}\left(m_{1}, r\right), G_{2}\left(m_{2}, r\right)\right)$. We verify that the simulator truthfully simulates the randomized encoding $\left(H_{1}, H_{2}\right)$ with deviation error of at most $\varepsilon$.

We begin with the case where $(x, y)$ is a 0 -input for $f$. For any $c$, let $L_{c}$ denote the distribution of the random variable $\left(G_{1}\left(F_{1}(x, c), r\right), G_{2}\left(F_{2}(y, c), r\right)\right)$ where $r \stackrel{R}{\leftarrow}\{0,1\}^{s(t(n))}$. Let $M$ denote the "mixture distribution" which is defined by first sampling $c \stackrel{R}{\leftarrow}\{0,1\}^{s(n)}$ and then outputting a random sample from $L_{c}$, that is, the distribution $M=\sum_{c \in\{0,1\}^{s(n)}} \operatorname{Pr}\left[U_{s(n)}=c\right] L_{c}$. Due to Lemma 3.1, we have that

$$
\Delta\left(\operatorname{Sim}_{g} ; M\right) \leq \sum_{c \in\{0,1\}^{s(n)}} \operatorname{Pr}\left[U_{s(n)}=c\right] \Delta\left(\operatorname{Sim}_{g} ; L_{c}\right)
$$

Let $C$ denote a subset of $c \in\{0,1\}^{s(n)}$ such that $\left(F_{1}(x, c), F_{2}(y, c)\right)$ is a 1-input for $g$. The set $C$ satisfies the following two properties: (1) $\forall c \in C \Delta\left(\operatorname{Sim}_{g} ; L_{c}\right) \leq \varepsilon(t(n))$ and $(2)|C| / 2^{s(n)} \geq 1-\delta(n)$. The property (1) holds because $G_{1}, G_{2}$ is private on 1 -inputs of $g$. The property (2) holds because $\operatorname{Dec}_{f}$ decodes correctly with the probability at least $1-\delta(n)$. After splitting the mixture sum in two, we have that

$$
\sum_{c \in\{0,1\}^{s(n)}} \operatorname{Pr}\left[U_{s(n)}=c\right] \Delta\left(\operatorname{Sim}_{g} ; L_{c}\right)=\sum_{c \in C} 2^{-s(n)} \Delta\left(\operatorname{Sim}_{g} ; L_{c}\right)+\sum_{c \notin C} 2^{-s(n)} \Delta\left(\operatorname{Sim}_{g} ; L_{c}\right) .
$$

Because of the properties of $C$, we have that the first sum is upperbounded by $\varepsilon(t(n))$ and the second one is upperbounded by $\delta(n)$. This implies that $\Delta\left(\operatorname{Sim}_{g} ; M\right) \leq \delta(n)+\varepsilon(t(n))$.

We move on to the case where $(x, y)$ is a 1 -input. Then

$$
\underset{c \stackrel{R}{\leftarrow}\{0,1\}^{s(n)}}{\Delta}\left(\operatorname{Sim}_{f} ;\left(F_{1}(x, c), F_{2}(y, c)\right)\right) \leq \varepsilon(n) .
$$

Consider the randomized procedure $G$ which, given $\left(m_{1}, m_{2}\right)$, samples $r \stackrel{R}{\leftarrow}\{0,1\}^{s(t(n))}$ and outputs the pair $\left(G_{1}\left(m_{1}, r\right), G_{2}\left(m_{2}, r\right)\right)$. Applying $G$ to the above distributions we get:

$$
\underset{(c, r) \stackrel{R}{\leftarrow}\{0,1\}^{u}}{\Delta}\left(G\left(\operatorname{Sim}_{f} ; r\right) ; G\left(F_{1}(x, c), F_{2}(y, c) ; r\right)\right) \leq \varepsilon(n) .
$$


Recall that, for a random $r \stackrel{R}{\leftarrow}\{0,1\}^{s(t(n)}$, it holds that $G\left(\operatorname{Sim}_{f} ; r\right) \equiv \operatorname{Sim}(1)$, and for every $r$, $G\left(F_{1}(x, c), F_{2}(y, c) ; r\right)=\left(H_{1}(x,(c, r)), H_{2}(y,(c, r))\right)$. Hence, Eq. 4 can be written as

$$
\underset{(c, r) \stackrel{R}{\leftarrow}\{0,1\}^{u}}{\Delta}\left(\operatorname{Sim}(1) ;\left(H_{1}(x,(c, r)), H_{2}(y,(c, r))\right)\right) \leq \varepsilon(n) .
$$

Since $\varepsilon(n) \leq \max (\varepsilon(n), \delta(n)+\varepsilon(t(n)))$, the theorem follows.

\section{Constructing CDS Schemes}

\subsection{From 1PSM to CDS}

In this section we construct a CDS scheme from a 1PSM protocol. Our construction is based on the observation (due to GIKM00]) that constructing a CDS scheme for a function $f$ can be reduced to constructing a PSM scheme for the sharing function $f^{\prime}((x, s),(y, s))=f(x, y) \wedge s$. We show that one can strengthen this statement by substituting PSM with a weaker security primitive 1PSM.

Theorem 2.5. Assume that the function $f$ has a $\delta$-complete $\varepsilon$-private $1 \mathrm{PSM}$ with communication complexity $t$ and randomness complexity s. Then the function $\bar{f}=1-f$ has a $\delta$-correct and $\varepsilon$-private CDS scheme with communication complexity $t$ and randomness complexity $s$.

Proof. Let $f:\{0,1\}^{n} \times\{0,1\}^{n} \rightarrow\{0,1\}$. Let $F_{1}, F_{2}:\{0,1\}^{n} \times\{0,1\}^{s} \rightarrow\{0,1\}^{t}$ be a $\delta$-correct and $\varepsilon$-private on 1-inputs 1PSM for $f$ with decoder $\operatorname{Dec}_{f}$ and simulator $\operatorname{Sim}_{f}$. Let $g$ denote $1-f$. Then, $\left(F_{1}, F_{2}\right)$ is $\delta$-correct and $\varepsilon$-private on 0-inputs 1PSM for $g$ with $\operatorname{Dec}_{g}=1-\operatorname{Dec}_{f}$ and $\operatorname{Sim}_{g}=\operatorname{Sim}_{f}$.

We construct a CDS scheme $\left(H_{1}, H_{2}\right)$ for $g$ as follows. Let $\left(x_{0}, y_{0}\right)$ be some fixed 0-input of $g$. We define $H_{1}(x, b, c)$ to output $F_{1}\left(x_{0}, c\right)$ if $b=0$, and $F_{1}(x, c)$ if $b=1$. Similarly, $H_{2}(y, b, c)$ outputs $F_{2}\left(y_{0}, c\right)$ if $b=0$ and $F_{2}(y, c)$ if $b=1$. The decoder Dec simply applies the 1PSM decoder of $g$, namely: given two messages $m_{1}$ and $m_{2}$, we reconstruct the secret $b$ by outputting $\operatorname{Dec}_{g}\left(m_{1}, m_{2}\right)$. We define the simulator Sim to run the simulator $\operatorname{Sim}_{g}$.

We prove that the pair $\left(H_{1}, H_{2}\right)$ is a CDS scheme for $g$.

$\delta$-Correctness: Take any 1-input $(x, y)$ of $g$ :

- If $b=0$ then $m_{1}=F_{1}\left(x_{0}, c\right)$ and $m_{2}=F_{2}\left(y_{0}, c\right)$. By the correctness property of 1PSM, we have that $\operatorname{Dec}_{g}\left(m_{1}, m_{2}\right)=\operatorname{Dec}_{g}\left(F_{1}\left(x_{0}, c\right), F_{2}\left(y_{0}, c\right)\right)=g\left(x_{0}, y_{0}\right)=0$ except with probability $\delta$.

- If $b=1$ then $m_{1}=F_{1}(x, c)$ and $m_{2}=F_{2}(y, c)$. By the correctness property of 1PSM, we have that $\operatorname{Dec}_{g}\left(m_{1}, m_{2}\right)=\operatorname{Dec}_{g}\left(F_{1}(x, c), F_{2}(y, c)\right)=g(x, y)=1$ except with probability $\delta$.

$\varepsilon$-PRIVACY: Fix some 0-input $(x, y)$ of $g$. Then, by the 1-sided privacy of the 1PSM, we have that, for $b=0$,

$$
\underset{c \leftarrow\{0,1\}^{s}}{\Delta}\left(\operatorname{Sim}(x, y) ;\left(H_{1}(x, 0, c), H_{2}(y, 0, c)\right)\right)=\underset{c \leftarrow}{\underset{c}{R}\{0,1\}^{s}} \underset{ }{\Delta}\left(\operatorname{Sim}_{g} ;\left(F_{1}\left(x_{0}, c\right), F_{2}\left(y_{0}, c\right)\right)\right) \leq \varepsilon,
$$

and, for $b=1$,

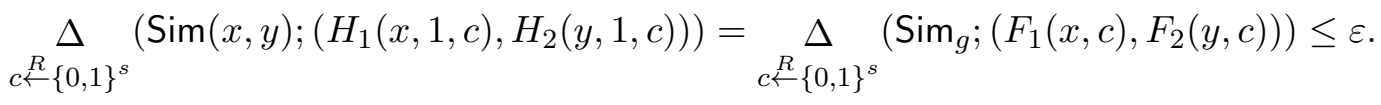




\subsection{From ZAM to CDS}

We now describe a direct construction of CDS from ZAM that avoids the overhead in the transformation from ZAM to 1PSM (Theorem 2.3). The saving is mainly due to the fact that, unlike the 1PSM setting, in the CDS setting the decoder is allowed to depend on the inputs $(x, y)$.

Theorem 2.6. Assume that the function $f:\{0,1\}^{n} \times\{0,1\}^{n} \rightarrow\{0,1\}$ has a $\delta$-sound ZAM protocol with communication complexity $\ell$ and randomness complexity $m$. Then the following hold.

1. The function $\bar{f}=1-f$ has a $\delta$-correct and 0 -private CDS with communication complexity $m$ and randomness complexity $2 m$.

2. For any $t \in \mathbb{N}$, the function $\bar{f}$ has $a\left(\delta+2^{-t}\right)$-correct and $\left(2^{-t}\right)$-private CDS with communication complexity $m$ and randomness complexity $(\ell+t)$.

Proof. Let $f:\{0,1\}^{n} \times\{0,1\}^{n} \rightarrow\{0,1\}$ be a function with a $\delta$-sound ZAM protocol $(A, B)$ with randomness complexity of $m$ and communication complexity of $\ell$. Fix some integer $k$. We start by recalling some notation from Theorem 2.3 . For a pair of inputs $(x, y)$ let

$$
E_{x y}:=\left\{\left(r_{A}, r_{B}\right) \in\{0,1\}^{m} \times\{0,1\}^{m} \mid \exists p: A\left(x, r_{A}, p\right)=1=B\left(y, r_{B}, p\right)\right\} .
$$

Let $\mathcal{A}_{p}^{x}:=\left\{r_{A} \in\{0,1\}^{m} \mid A\left(x, r_{A}, p\right)=1\right\}$ and $\mathcal{B}_{p}^{y}:=\left\{r_{B} \in\{0,1\}^{m} \mid B\left(y, r_{B}, p\right)=1\right\}$.

We construct a CDS scheme $\left(F_{1}, F_{2}\right)$ for $g$ as follows. As common randomness the scheme takes $p$ sampled from the accepting distribution $D_{\mathrm{ACC}}$ of the ZAM scheme (as in Theorem 2.3, $D_{\mathrm{ACC}}$ can be perfectly simulated using $2 m$ uniform bits). On an input $(x, b, p)$ the function $F_{1}$ computed by Alice outputs $r_{1} \stackrel{R}{\leftarrow}\{0,1\}^{m}$ if $b=1$, and $r_{1} \stackrel{R}{\leftarrow} \mathcal{A}_{p}^{x}$, otherwise. Similarly, on an input $(y, b, p)$

the function $F_{2}$ computed by Bob outputs $r_{2} \stackrel{R}{\leftarrow}\{0,1\}^{m}$ if $b=1$, and $r_{2} \stackrel{R}{\leftarrow} \mathcal{B}_{p}^{y}$, otherwise. The decoding procedure works as follows: on input $\left(x, y, r_{1}, r_{2}\right)$ the decoder outputs 0 if $\left(r_{1}, r_{2}\right) \in E_{x y}$, and 1 otherwise.

Now we prove that $\left(F_{1}, F_{2}\right)$ is a CDS scheme for $\bar{f}$ by verifying its security properties:

$\delta$-Correctness: Take any 1 -input $(x, y)$ of $\bar{f}$, which is a 0 -input of $f$.

- If the secret bit $b=0$, then $r_{1}$ and $r_{2}$ are sampled uniformly from $\mathcal{A}_{p}^{x}$ and $\mathcal{B}_{p}^{y}$, respectively. This means that with probability 1 the pair $\left(r_{1}, r_{2}\right)$ lands in $E_{x y}$ and hence decoding of $\left(x, y, r_{1}, r_{2}\right)$ never fails in this case.

- If the secret bit $b=1$, then $r_{1}$ and $r_{2}$ are sampled uniformly from $\{0,1\}^{m}$. This implies that the probability that $\left(x, y, r_{1}, r_{2}\right)$ is decoded incorrectly to 0 is the probability of $\left(r_{1}, r_{2}\right)$ landing in $E_{x y}$. Due to the soundness property of ZAM, the latter probability is at most $\delta$.

Perfect Privacy: We define the simulator Sim to output a random point $\left(r_{1}, r_{2}\right) \in\{0,1\}^{m} \times$ $\{0,1\}^{m}$. Take any 0 -input $(x, y)$ of $\bar{f}$, which is a 1 -input of $f$. We verify that Sim perfectly simulates the distribution of $\left(F_{1}, F_{2}\right)$ for any $b \in\{0,1\}$. For $b=0$ we have that $F_{1}$ and $F_{2}$ each output $U_{m}$ by construction. For $b=1$ we use the observation from the proof of Theorem 2.3 that the joint distribution of $\left(r_{1}, r_{2}\right)$ sampled from $\mathcal{A}_{p}^{x}$ and $\mathcal{B}_{p}^{y}$ for $p \stackrel{R}{\leftarrow} D_{\mathrm{ACC}}$ is uniform over $\{0,1\}^{m} \times\{0,1\}^{m}$.

The second protocol. Similarly to Theorem 2.3 , the second protocol is identical to the first protocol except it uses an approximation of $D_{\mathrm{ACC}}$. We know that for any $t \in \mathbb{N}$ the distribution $D_{\mathrm{ACC}}$ can be approximated using $(\ell+t)$ bits at the cost of deviating by $2^{-t}$ in terms of the statistical distance from $D_{\mathrm{ACC}}$. This introduces an additional error of $2^{-t}$ in both privacy and correctness of the second protocol. 


\subsection{CDS for Dependency Programs}

A dependency program is a model of computation introduced in PS98. The original model captures functions over binary inputs.

Definition 8.1 (DP). A dependency program over a field $\mathbb{F}$ is a pair $(M, \rho)$, where $M$ is a matrix over $\mathbb{F}$ and $\rho$ is a labeling of the rows of $M$ by the literals from $\left\{x_{1}, \ldots, x_{n}, \bar{x}_{1}, \ldots, \bar{x}_{n}\right\}$ (every row is labeled with a single literal, and the same literal can be used in many rows). For an input $u \in\{0,1\}^{n}$ let $M_{u}$ denote the matrix obtained from $M$ by selecting only the rows assigned to the literals satisfied by $u$, i.e., a row labeled with $x_{i}$ (resp. $\bar{x}_{i}$ ) is chosen if the $i^{\text {th }}$ bit of $u$ is 1 (resp., $0)$. A dependency program accepts an input $u$ if and only if the rows of $M_{u}$ are linearly dependent. A dependency program computes a Boolean function $f$ if it accepts only 1-inputs of $f$. The size of the dependency program is the number of rows in $M$. We also write $|M|$ to denote the number of row the matrix $M$ has.

The number of columns $s$ in DP is not counted towards its size. Without loss of generality we may assume that $s$ is upper-bounded by the number of rows (the size) since the matrix $M$ can be restricted to a maximal set of linearly independent columns without changing the function that is computed (cf. [BG99, Remark 2.4]). It will also be convenient to assume that the number of rows labeled by $x_{i}$ is equal to the number of rows that are labeled by its complement $\bar{x}_{i}$. (If this is not the case and $M_{x_{i}}$ contains less rows than $M_{\bar{x}_{i}}$ then we can add new linearly independent rows labeled by $x_{i}$, possibly at the expense of increasing the number of columns. Overall, the size of the resulting dependency program will be at most twice as large as the size of the original program.) Observe that if the input is partitioned between Alice and Bob, then the above convention guarantees that for every input $x$ (resp., $y$ ) Alice (resp., Bob) will hold a matrix $M_{x}$ (resp., $M_{y}$ ) with a fixed number of rows which is independent of the input.

We construct CDS for dependency programs. The following theorem generalizes Theorem 2.7 from the introduction to arbitrary finite fields.

Theorem 8.2 (Theorem 2.7 generalized). Assume that the function $f:\{0,1\}^{n} \times\{0,1\}^{n} \rightarrow$ $\{0,1\}$ has a dependency program of size $m$ over a finite field $\mathbb{F}$. Then, for every $t \in \mathbb{N}$, the function $f$ has an $(1 /|\mathbb{F}|)^{t}$-correct perfectly-private CDS scheme where the secret is an element of $\mathbb{F}$ and the protocol communicates $O(m \cdot t)$ field elements and consumes $O(m \cdot t)$ random field elements.

Note that for large fields, the scheme achieves low decoding error even for small values of $t$ (e.g., 1).

Proof. Let $(M, \rho)$ be a dependency program for the function $f:\{0,1\}^{n} \times\{0,1\}^{n} \rightarrow\{0,1\}$ over the finite field $\mathbb{F}$. Let $s$ denote the number of columns in $M$, and let $m_{1}$ (resp., $m_{2}$ ) denote the number of rows of $M$ held by Alice for an input $x$ (resp., held by Bob for an input $y$ ). Recall that, by convention, $m_{1}$ and $m_{2}$ are independent of $x$ and $y$, and that $m^{\prime}=m_{1}+m_{2}$ is at most $m$, the size of $M$.

We present a basic CDS scheme $\left(F_{1}, F_{2}\right)$ for $f$ where the secret $b$ can be an arbitrary field element. The scheme communicates at most $2 m$ field elements, and uses at most $2 m$ random field elements. It achieves perfect privacy and has a completeness error of $1 /|\mathbb{F}|$. In fact, the decoder will either output the right answer or will output, with probability $1 /|\mathbb{F}|$, a special failure symbol. Therefore, by repeating the protocol $t$ times (with independent randomness), we can reduce the 
error to $|\mathbb{F}|^{-t}$ with a multiplicative overhead of $t$ in communication and randomness, as stated in the theorem.

The basic CDS scheme $\left(F_{1}, F_{2}\right)$ is defined as follows. As common randomness the scheme takes a pair of random vectors $c \in \mathbb{F}^{s}$ and $d \in \mathbb{F}^{m^{\prime}}$. On an input $(x, b, c, d)$, the function $F_{1}$ computed by Alice outputs the pair $\left(d_{1}, r_{1}\right)$ where $d_{1} \in \mathbb{F}^{m_{1}}$ is the first $m_{1}$ entries of the vector $d$, and $r_{1}=M_{x} \cdot c+b \cdot d_{1}$. (Recall that $b \in \mathbb{F}$ is a scalar.) Similarly, on an input $(y, b, c, d)$ the function $F_{2}$ computed by Bob outputs the pair $\left(d_{2}, r_{2}\right)$ where $d_{2} \in \mathbb{F}^{m_{2}}$ is the last $m_{2}$ entries of the vector $d$ and $r_{2}=M_{y} \cdot c+b \cdot d_{2}$. For a 1-instance $(x, y)$, the decoding procedure decodes $\left(d=\left(d_{1}, d_{2}\right), r=\left(r_{1}, r_{2}\right)\right)$ as follows: (1) The decoder finds a non-zero vector $v \in \mathbb{F}^{m^{\prime}}$ for which $v^{T} M_{x y}=\mathbf{0}$ (such a vector must exist since the rows of $M_{x y}$ are linearly dependent); (2) If the dot product $\left(v^{T} \cdot d\right)$ is non-zero the decoder outputs the value $b^{\prime}=\left(v^{T} \cdot r\right) /\left(v^{T} \cdot d\right)$, and otherwise it outputs a special failure symbol.

We prove that the pair $\left(F_{1}, F_{2}\right)$ is a CDS for $f$.

Correctness: Fix some 1-input $(x, y)$ of $f$. Since $v$ is in the left nullspace of $M_{x y}$, it holds that

$$
v^{T} \cdot r=v^{T}\left(M_{x y} \cdot c+b \cdot d\right)=b \cdot\left(v^{T} \cdot d\right) .
$$

Therefore, decoding succeeds as long as $\left(v^{T} \cdot d\right) \neq 0$. The latter event happens with probability $1-1 /|\mathbb{F}|$ since $d \in \mathbb{F}^{m^{\prime}}$ is uniformly distributed.

Perfect Privacy: Fix some 0-input $(x, y)$ of $f$. We show that in this case the random variables $\left(d_{1}, r_{1}\right)=F_{1}(x, b, c, d)$ and $\left(d_{2}, r_{2}\right)=F_{2}(y, b, c, d)$ induced by a random choice of $c$ and $d$, are just vectors of uniformly and independently chosen field elements. First note that, by construction, $d=\left(d_{1}, d_{2}\right)$ is uniformly chosen from $\mathbb{F}^{m^{\prime}}$. Recall that $r=M_{x y} \cdot c+b \cdot d$, and therefore it suffices to show that $M_{x y} \cdot c$ is uniform over $\mathbb{F}^{m^{\prime}}$. The latter boils down to showing that the image of $M_{x y}$ is equal to $\mathbb{F}^{m^{\prime}}$. Indeed, since $(x, y)$ is 0 -input of $f$, the rows of the matrix $M_{x y}$ are linearly independent (i.e., the left nullspace of $M_{x y}$ has rank 0), and so, by the fundamental theorem of linear algebra, the linear space spanned by the columns of $M_{x y}$ equals to $\mathbb{F}^{m^{\prime}}$.

Remark 8.3 (Linearity). We say that a CDS $\left(F_{1}, F_{2}\right)$ is linear GKW15] if for any fixed 1-input $(x, y)$ the decoding function Dec $_{x, y}$ which maps the messages of Alice and Bob (viewed together as a vector over a field $\mathbb{F}$ ) to the secret $b \in \mathbb{F}$ is linear over $\mathbb{F}$. It is not hard to verify that Theorem 2.7 yields a linear CDS. In fact, our scheme satisfies a stronger notion of linearity: for any fixed input $(x, y)$ the functions $F_{1}$ and $F_{2}$ are degree 1 functions in the secret $b$ and in the common randomness randomness $(c, d)$. These linearity properties are useful for some applications such as Attribute Based Encryption schemes (cf. [IW14, GKW15]).

Remark 8.4 (Extension to non-binary inputs). We can get CDS for functions whose inputs are field elements, i.e., $f: \mathbb{F}^{n} \times \mathbb{F}^{n} \rightarrow\{0,1\}$, by considering an arithmetic generalization of dependencies programs. Formally, we define an arithmetic dependency program (ADP) over a field $\mathbb{F}$ to be a triplet $(W, V, \rho)$, where $W, V \in \mathbb{F}^{m \times s}$ and $\rho:[m] \rightarrow[n]$. For an input $u \in \mathbb{F}^{n}$, let $M_{u}$ denote the $m \times s$ matrix whose $i$-th row corresponds to $W_{i} \cdot u_{\rho(i)}+V_{i}$, where $W_{i}$ and $V_{i}$ denote the $i$-th row of $W$ and $V$, respectively. An ADP computes a Boolean function $f$ if for every $u \in \mathbb{F}^{n}$ we have $f(u)=1$ if and only if the rows of $M_{u}$ are linearly dependent. Theorem 8.2 and its proof readily extends to ADPs. More generally, the CDS construction from Theorem 8.2 applies as long as Alice and Bob can locally compute matrices $M_{x}$ and $M_{y}$ (respectively) with the property that $f(x, y)=1$ if and only if the rows of the matrix $M=\left(\begin{array}{l}M_{x} \\ M_{y}\end{array}\right)$ are linearly dependent. 
Remark 8.5 (Comparison to CDS for span-programs). It is instructive to compare our construction to the CDS construction of span programs (implicit in [IW14, Section 7]). Say that Alice's input $x$ defines a set of row vectors which together form the matrix $M_{x}$, and that Bob's input $y$ defines a set of row vectors which together form the matrix $M_{y}$. For span program the predicate accepts $(x, y)$ if some target row vector $t \in \mathbb{F}^{s}$ is in the row-span of the $m \times s$ matrix $M=\left(\begin{array}{l}M_{x} \\ M_{y}\end{array}\right)$. (At the extreme, the rows of $M$ span the whole space.) To get a CDS, Alice and Bob use a shared random column vector $c \in \mathbb{F}^{s}$ and output the vector $M c=\left(\begin{array}{c}M_{x} c \\ M_{y} c\end{array}\right)$ together with $b+t c$ (i.e., the secret $b$ is masked by the dot-product of $t$ and $c)$. In a more compact form, the CDS can be written as

$$
\left(\begin{array}{c}
M \\
t
\end{array}\right) \cdot c+b^{\prime}
$$

where $b^{\prime}=(0 \cdots 0 b)$. It is not hard to show that this is a valid CDS. (Indeed, if $t$ can be written as a linear combination of the rows of $M$, i.e., $t=w^{\top} M$, then the referee can recover $t c=w^{\top} M c$ and un-pad the secret; On the other hand, if $t$ is not spanned by the rows of $M$ then tc is uniformly distributed conditioned on $M c$, and so the secret is perfectly hidden.) Getting back to dependency programs, recall that a DP is satisfied if the rows of $M$ are linearly dependent (at the extreme no vector is spanned by the rows of $M$ ), which intuitively correspond to the converse of span programs. Indeed, the PSM for dependency programs can be written as

$$
\left(\begin{array}{ll}
M & d
\end{array}\right) \cdot\left(\begin{array}{l}
c \\
b
\end{array}\right)
$$

where $c \in \mathbb{F}^{s}$ and $d \in \mathbb{F}^{m}$ are shared random inputs. Comparing (5) to (6), we see that for span programs the secret is padded by a random image $v$ of (an extended version of) $M$, whereas for dependency programs the secret is hidden as part of the preimage of $v$. In this sense, the two constructions are dual to each other.

\section{Acknowledgement}

We thank Yuval Ishai and Hoeteck Wee for useful discussions about [IW14. We also thank Yuval Ishai for pointing out that our CDS construction for arithmetic branching programs extends to dependency programs.

\section{References}

[AIK06] B. Applebaum, Y. Ishai, and E. Kushilevitz. Cryptography in $\mathrm{NC}^{0}$. SIAM J. Comput., 36(4):845-888, 2006.

[AIK14] B. Applebaum, Y. Ishai, and E. Kushilevitz. How to garble arithmetic circuits. SIAM J. Comput., 43(2):905-929, 2014.

[AIK15] B. Applebaum, Y. Ishai, and E. Kushilevitz. Minimizing locality of one-way functions via semi-private randomized encodings. Electronic Colloquium on Computational Complexity (ECCC), 22:45, 2015. 
[AR16] B. Applebaum and P. Raykov. From private simultaneous messages to zero-information arthur-merlin protocols and back. In E. Kushilevitz and T. Malkin, editors, Theory of Cryptography - 13th International Conference, TCC 2016-A, Tel Aviv, Israel, January 10-13, 2016, Proceedings, Part II, volume 9563 of Lecture Notes in Computer Science, pages 65-82. Springer, 2016. Available as eprint report 2015/1046 at http://eprint. iacr.org/2015/1046.

[BFS86] L. Babai, P. Frankl, and J. Simon. Complexity classes in communication complexity theory (preliminary version). In 27th Annual Symposium on Foundations of Computer Science, Toronto, Canada, 27-29 October 1986, pages 337-347. IEEE Computer Society, 1986.

[BG99] A. Beimel and A. Gál. On arithmetic branching programs. J. Comput. Syst. Sci., 59(2):195-220, 1999.

[BIKK14] A. Beimel, Y. Ishai, R. Kumaresan, and E. Kushilevitz. On the cryptographic complexity of the worst functions. In Y. Lindell, editor, Theory of Cryptography - 11th Theory of Cryptography Conference, TCC 2014, San Diego, CA, USA, February 24-26, 2014. Proceedings, volume 8349 of Lecture Notes in Computer Science, pages 317-342. Springer, 2014.

[BM88] L. Babai and S. Moran. Arthur-merlin games: A randomized proof system, and a hierarchy of complexity classes. J. Comput. Syst. Sci., 36(2):254-276, 1988.

[CKGS98] B. Chor, E. Kushilevitz, O. Goldreich, and M. Sudan. Private information retrieval. J. $A C M, 45(6): 965-981,1998$.

[FKN94] U. Feige, J. Kilian, and M. Naor. A minimal model for secure computation (extended abstract). In F. T. Leighton and M. T. Goodrich, editors, Proceedings of the TwentySixth Annual ACM Symposium on Theory of Computing, 23-25 May 1994, Montréal, Québec, Canada, pages 554-563. ACM, 1994.

[GIKM00] Y. Gertner, Y. Ishai, E. Kushilevitz, and T. Malkin. Protecting data privacy in private information retrieval schemes. J. Comput. Syst. Sci., 60(3):592-629, 2000.

[GKW15] R. Gay, I. Kerenidis, and H. Wee. Communication complexity of conditional disclosure of secrets and attribute-based encryption. In R. Gennaro and M. Robshaw, editors, Advances in Cryptology - CRYPTO 2015 - 35th Annual Cryptology Conference, Santa Barbara, CA, USA, August 16-20, 2015, Proceedings, Part II, volume 9216 of Lecture Notes in Computer Science, pages 485-502. Springer, 2015.

[GPW15] M. Göös, T. Pitassi, and T. Watson. Zero-information protocols and unambiguity in arthur-merlin communication. In T. Roughgarden, editor, Proceedings of the 2015 Conference on Innovations in Theoretical Computer Science, ITCS 2015, Rehovot, Israel, January 11-13, 2015, pages 113-122. ACM, 2015.

[IK97] Y. Ishai and E. Kushilevitz. Private simultaneous messages protocols with applications. In Proceedings of the 5th Israeli Symposium on Theory of Computing and Systems, pages 174-183, June 1997. 
[IK00] Y. Ishai and E. Kushilevitz. Randomizing polynomials: A new representation with applications to round-efficient secure computation. In 41st Annual Symposium on Foundations of Computer Science, FOCS 2000, 12-14 November 2000, Redondo Beach, California, USA, pages 294-304. IEEE Computer Society, 2000.

[IK02] Y. Ishai and E. Kushilevitz. Perfect constant-round secure computation via perfect randomizing polynomials. In P. Widmayer, F. T. Ruiz, R. M. Bueno, M. Hennessy, S. Eidenbenz, and R. Conejo, editors, Automata, Languages and Programming, 29th International Colloquium, ICALP 2002, Malaga, Spain, July 8-13, 2002, Proceedings, volume 2380 of Lecture Notes in Computer Science, pages 244-256. Springer, 2002.

[Ish13] Y. Ishai. Randomization techniques for secure computation. In M. Prabhakaran and A. Sahai, editors, Secure Multi-Party Computation, volume 10 of Cryptology and Information Security Series, pages 222-248. IOS Press, 2013.

[IW14] Y. Ishai and H. Wee. Partial garbling schemes and their applications. In J. Esparza, P. Fraigniaud, T. Husfeldt, and E. Koutsoupias, editors, Automata, Languages, and Programming - 41st International Colloquium, ICALP 2014, Copenhagen, Denmark, July 8-11, 2014, Proceedings, Part I, volume 8572 of Lecture Notes in Computer Science, pages 650-662. Springer, 2014.

[Kla03] H. Klauck. Rectangle size bounds and threshold covers in communication complexity. In 18th Annual IEEE Conference on Computational Complexity (Complexity 2003), 7-10 July 2003, Aarhus, Denmark, pages 118-134. IEEE Computer Society, 2003.

[Kla10] H. Klauck. A strong direct product theorem for disjointness. In L. J. Schulman, editor, Proceedings of the 42nd ACM Symposium on Theory of Computing, STOC 2010, Cambridge, Massachusetts, USA, 5-8 June 2010, pages 77-86. ACM, 2010.

[KW93] M. Karchmer and A. Wigderson. On span programs. In Proceedings of the Eigth Annual Structure in Complexity Theory Conference, San Diego, CA, USA, May 18-21, 1993, pages 102-111. IEEE Computer Society, 1993.

[PS98] P. Pudlák and J. Sgall. Algebraic models of computation and interpolation for algebraic proof systems. In Proof Complexity and Feasible Arithmetic, volume 39 of DIMACS Series in Discrete Mathematics and Theor. Comput. Sci., pages 279-296. Am. Math. Soc., Providence, RI, 1998.

\section{A [FKN94] is pPSM}

We show that the PSM construction given in FKN94 is also perfect, i.e., is a pPSM construction. We start by describing the [FKN94] scheme $\left(F_{1}, F_{2}\right)$ for a boolean $f:[N] \times[N] \rightarrow\{0,1\}$. (Our description is based on [BIKK14.) The shared randomness of the scheme is $N$ random bits $r=$ $\left(r_{0}, \ldots, r_{N-1}\right)$ and a random index $s \in[N]$. The algorithms $F_{1}$ and $F_{2}$ are defined as follows: 
Algorithm $F_{1}(x,(r, s))$ :

1. For each $i \in\{0, \ldots, N-1\}$, compute $c_{i}=f(x, i)+r_{i}$.

2. For each $i \in\{0, \ldots, N-1\}$, compute $d_{i}=c_{i+s} \bmod N$.

3. Output $m_{1}=\left(d_{0}, \ldots, d_{N-1}\right)$.
Algorithm $F_{2}(y,(r, s))$ :

1. Compute $u=r_{y}$ and $v=y-s$.

2. Output $m_{2}=(u, v)$.

Decoding is performed by outputting the value $u+d_{v}$. We have that $F_{1}$ 's output space is $R_{1}=\{0,1\}^{N}$ and the output space of $F_{2}$ is $R_{2}=\{0,1\} \times[N]$. Define $F_{x y}(r, s):\{0,1\}^{N-1} \times[N] \rightarrow$ $R_{1} \times R_{2}$ by $\left(F_{1}(x,(r, s)), F_{2}(y,(r, s))\right)$. For $b \in\{0,1\}$, let $S_{b}:=\left\{F_{x y}(r, s) \mid(r, s) \in\{0,1\}^{N} \times\right.$ $\left.[N],(x, y) \in f^{-1}(b)\right\}$. We prove that $S_{0}$ and $S_{1}$ satisfy the required combinatorial properties of pPSM (Remark 4.3):

- $S_{0} \cap S_{1}=\emptyset$, since each $S_{b}$ consists of $\left(\left(d_{0}, \ldots, d_{N-1}\right),(u, v)\right)$ such that $b=u+d_{v}$.

- $S_{0} \cup S_{1}=R_{1} \times R_{2}$, since $S_{b}$ consists of all pairs $\left(\left(d_{0}, \ldots, d_{N-1}\right),(u, v)\right)$ satisfying the correctness requirement $b=u+d_{v}$.

- For any fixed input $(x, y)$, we show that $F_{x y}$ is a bijection on $S_{f(x, y)}$. First, we prove that it is an injection. Indeed, say that $F_{x y}(r, s)=F_{x y}\left(r^{\prime}, s^{\prime}\right)=(d, u, v)$ and let $c=\left(c_{1}, \ldots, c_{N-1}\right)$ and $c^{\prime}=\left(c_{1}^{\prime}, \ldots, c_{N-1}^{\prime}\right)$ denote the intermediate values computed by $F_{1}(x,(r, s))$ and $F_{1}\left(x,\left(r^{\prime}, s^{\prime}\right)\right)$, respectively. Then, by correctness, it holds that $y-s=y-s^{\prime}$. This means that $s=s^{\prime}$, which, in turn, implies that $c=c^{\prime}$ and so $r=r^{\prime}$. It follows that $F_{x y}$ is injective. Second, we claim that $F_{x y}$ is a surjection on $S_{f(x, y)}$. Towards this end, we show that any tuple $\left(\left(d_{0}, \ldots, d_{N-1}\right),(u, v)\right)$ from $S_{f(x, y)}$ has a preimage $(s, r)$ under $F_{x y}$. Indeed, let $s:=y-v$, and for each $i \in\{0, \ldots, N-1\}$, let $r_{i}:=d_{i-s \bmod N}-f(x, i)$. By construction, we have that $(r, s)$ is a preimage of $\left(\left(d_{0}, \ldots, d_{N-1}\right),(u, v)\right)$, i.e., $F_{x y}(r, s)$ equals to $\left(d_{0}, \ldots, d_{N-1}\right),(u, v)$.

\section{B [BIKK14] is pPSM}

In this Section we describe the PSM protocol of [BIKK14] and prove that it forms a pPSM. To simplify the proof, our description slightly deviates from the original description though the resulting protocol is identical.

Before constructing a pPSM for the function $f:[N] \times[N] \rightarrow\{0,1\}$, we introduce some auxiliary definitions. Let us view $f$ as a function of four arguments by splitting its first and second argument in half, i.e., $f(x, y)=f\left(v_{1}, v_{2}, v_{3}, v_{4}\right)$ where each $v_{i} \in\left[N^{1 / 2}\right]$. Following [BIKK14], we associate $f$ with a 4-dimensional cube as follows: each coordinate $\left(v_{1}, v_{2}, v_{3}, v_{4}\right)$ of the cube is associated with the value $f\left(v_{1}, v_{2}, v_{3}, v_{4}\right)$. For any $v \in\left[N^{1 / 2}\right]$, let $\boldsymbol{e}_{v}$ denote the $N^{1 / 2}$-bit indicator vector which has 1 at location $v$, and 0 elsewhere. For any two $N^{1 / 2}$-bit vectors $u_{1}, u_{2}$ let $\left\langle u_{1}, u_{2}\right\rangle$ denote their inner product. For four $N^{1 / 2}$-bit vectors $u_{1}, u_{2}, u_{3}, u_{4}$ we define the function $G\left(u_{1}, u_{2}, u_{3}, u_{4}\right)$ to compute the XOR of the subcube defined by the 1 -coordinates in $u_{1}, u_{2}, u_{3}, u_{4}$, i.e.,

$$
G\left(u_{1}, u_{2}, u_{3}, u_{4}\right)=\sum_{v_{1}, v_{2}, v_{3}, v_{4} \in\left[N^{1 / 2}\right]}\left\langle\boldsymbol{e}_{v_{1}}, u_{1}\right\rangle \cdot\left\langle\boldsymbol{e}_{v_{2}}, u_{2}\right\rangle \cdot\left\langle\boldsymbol{e}_{v_{3}}, u_{3}\right\rangle \cdot\left\langle\boldsymbol{e}_{v_{4}}, u_{4}\right\rangle \cdot f\left(v_{1}, v_{2}, v_{3}, v_{4}\right),
$$

where addition and multiplication are computed over the binary field. The following fact has been observed in CKGS98. 
Fact B.1. Let $v_{1}, v_{2}, v_{3}, v_{4} \in\left[N^{1 / 2}\right]$ be any four values and let $u_{1}, u_{2}, u_{3}, u_{4}$ be any four $N^{1 / 2}$-bit vectors. For each $k \in[4]$, let $u_{k}^{0}=u_{k}$ and let $u_{k}^{1}=u_{k} \oplus \boldsymbol{e}_{v_{k}}$ (where $\oplus$ is a bit-wise XOR of vectors). Then, it holds that

$$
f\left(v_{1}, v_{2}, v_{3}, v_{4}\right)=\sum_{\left(b_{1}, b_{2}, b_{3}, b_{4}\right) \in\{0,1\}^{4}} G\left(u_{1}^{b_{1}}, u_{2}^{b_{2}}, u_{3}^{b_{3}}, u_{4}^{b_{4}}\right) .
$$

We now describe how Alice and Bob each holding input $x=\left(x_{1}, x_{2}\right)$ and $y=\left(y_{1}, y_{2}\right)$, respectively, compute an encoding of $f\left(x_{1}, x_{2}, y_{1}, y_{2}\right)$. Let $u_{1}, u_{2}, u_{3}, u_{4}$ be four random $N^{1 / 2}$-bit vectors that Alice and Bob use as common randomness. For $k \in[4]$ and $b \in\{0,1\}$, define additional vectors $u_{k}^{b}$ as follows: for each $k \in[4]$, let $u_{k}^{0}=u_{k}$; and let $u_{1}^{1}=u_{1} \oplus \boldsymbol{e}_{x_{1}}, u_{2}^{1}=u_{2} \oplus \boldsymbol{e}_{x_{2}}, u_{3}^{1}=u_{3} \oplus \boldsymbol{e}_{y_{1}}$, $u_{4}^{1}=u_{4} \oplus \boldsymbol{e}_{y_{2}}$. Alice and Bob compute the encoding of $f\left(x_{1}, x_{2}, y_{1}, y_{2}\right)$ by computing an encoding of the sum $\sum_{\left(b_{1}, b_{2}, b_{3}, b_{4}\right) \in\{0,1\}^{4}} G\left(u_{1}^{b_{1}}, u_{2}^{b_{2}}, u_{3}^{b_{3}}, u_{4}^{b_{4}}\right)$. They do this by splitting the sum into two summands:

$$
G\left(u_{1}^{1}, u_{2}^{1}, u_{3}^{1}, u_{4}^{1}\right)+\sum_{\left(b_{1}, b_{2}, b_{3}, b_{4}\right) \in\{0,1\}^{4} \backslash\{(1,1,1,1)\}} G\left(u_{1}^{b_{1}}, u_{2}^{b_{2}}, u_{3}^{b_{3}}, u_{4}^{b_{4}}\right) .
$$

The first summand is encoded with the tuple $\left(u_{1}^{1}, u_{2}^{1}, u_{3}^{1}, u_{4}^{1}\right)$, i.e., the values $u_{i}^{1}$ are sent by Alice and Bob in plain, so that the Referee can compute the term $G\left(u_{1}^{1}, u_{2}^{1}, u_{3}^{1}, u_{4}^{1}\right)$. As observed in BIKK14, the second summand can be computed by a multiplicative depth-1 circuit $C\left(x, y,\left(u_{1}, u_{2}, u_{3}, u_{4}\right)\right)$ of size $O\left(N^{1 / 2}\right)$ and so it can be encoded via the following lemma whose proof is implicit in [K02] (see also [AIK14]).

Lemma B.2. Every multiplicative depth-1 circuit of size $m$ has a pPSM with randomness and communication complexity of size $O(m)$.

Let $H_{1}$ and $H_{2}$ denote the pPSM of the circuit $C$. Then, the encoding of the second term is computed by Alice and Bob by applying $H_{1}$ and $H_{2}$, respectively.

Formally, the resulting encoding $F_{1}, F_{2}$ for the function $f$ is described below.

Parameters: Alice's input $x=\left(x_{1}, x_{2}\right)$, Bob's input $y=\left(y_{1}, y_{2}\right)$, four random $N^{1 / 2}$-bit vectors $\left(u_{1}, u_{2}, u_{3}, u_{4}\right)=u$ and a common randomness $c$ used by $H_{1}, H_{2}$.

Alice's algorithm $F_{1}(x,(u, c))$ :

1. Compute $m_{1}=H_{1}((x, u), c)$.

2. Compute $u_{1}^{1}=u_{1} \oplus \boldsymbol{e}_{x_{1}}$ and $u_{2}^{1}=u_{2} \oplus \boldsymbol{e}_{x_{2}}$.

3. Output $M_{1}=\left(u_{1}^{1}, u_{2}^{1}, m_{1}\right)$.
Bob's algorithm $F_{2}(y,(u, c))$ :

1. Compute $m_{2}=H_{2}((y, u), c)$.

2. Compute $u_{3}^{1}=u_{3} \oplus \boldsymbol{e}_{y_{1}}$ and $u_{4}^{1}=u_{4} \oplus \boldsymbol{e}_{y_{2}}$.

3. Output $M_{2}=\left(u_{3}^{1}, u_{4}^{1}, m_{2}\right)$.

The decoding algorithm works as follows: Given $\left(u_{1}^{1}, u_{2}^{1}, m_{1}\right)$ and $\left(u_{3}^{1}, u_{4}^{1}, m_{2}\right)$, the decoder first uses a decoder of the pPSM $\left(H_{1}, H_{2}\right)$ to recover the bit $z=C(x, y, u)$, and then outputs $z+$ $G\left(u_{1}^{1}, u_{2}^{1}, u_{3}^{1}, u_{4}^{1}\right)$.

Lemma B.3. The encoding $\left(F_{1}, F_{2}\right)$ is a pPSM for $f$ with randomness and communication complexity of $O\left(N^{1 / 2}\right)$.

Proof. We prove that $\left(F_{1}, F_{2}\right)$ is a pPSM by viewing it as a composition of two perfect encodings (see Remark 4.5). Consider the encoding $J$, which takes $(x, y)$ as deterministic inputs and $u=$ $\left(u_{1}, u_{2}, u_{3}, u_{4}\right)$ where $u_{i} \in\{0,1\}^{N^{1 / 2}}$ as common randomness, and outputs the pair

$$
\left(C(x, y, u),\left(u_{1}^{1}, u_{2}^{1}, u_{3}^{1}, u_{4}^{1}\right)\right) \in\{0,1\} \times\left[N^{1 / 2}\right]^{4},
$$


where each $u_{k}^{1}$ are computed as in $\left(F_{1}, F_{2}\right)$. We prove that $J(x, y, u)$ is a pPSM of $f$ by showing that $J$ satisfies the required combinatorial properties of pPSM as formulated in Remark 4.3, Let $J_{x y}(u)=J(x, y, u)$ and, for $b \in\{0,1\}$, define $S_{b}:=\left\{J_{x y}(u) \mid u \in\left[N^{1 / 2}\right]^{4},(x, y) \in f^{-1}(b)\right\}$. Then the following hold:

- $S_{0} \cap S_{1}=\emptyset$ since each $S_{b}$ consists of $\left(d,\left(w_{1}, w_{2}, w_{3}, w_{4}\right)\right)$ such that $d+G\left(w_{1}, w_{2}, w_{3}, w_{4}\right)=b$.

- We prove that $S_{0} \cup S_{1}=\{0,1\} \times\left[N^{1 / 2}\right]^{4}$. Take any $\left(d,\left(w_{1}, w_{2}, w_{3}, w_{4}\right)\right)$ from $\{0,1\} \times\left[N^{1 / 2}\right]^{4}$. Let $z=d+G\left(w_{1}, w_{2}, w_{3}, w_{4}\right)$. Take any $z$-input $(x, y)$ of $f$. Define $u_{1}=w_{1} \oplus \boldsymbol{e}_{x_{1}}, u_{2}=$ $w_{2} \oplus \boldsymbol{e}_{x_{2}}, u_{3}=w_{3} \oplus \boldsymbol{e}_{y_{1}}$, and $u_{4}=w_{4} \oplus \boldsymbol{e}_{y_{2}}$. By construction we have that $J_{x y}\left(u_{1}, u_{2}, u_{3}, u_{4}\right)=$ $\left(d,\left(w_{1}, w_{2}, w_{3}, w_{4}\right)\right)$.

- For any fixed input $(x, y)$, we show that $J_{x y}$ is a bijection on $S_{f(x, y)}$. First, we prove that it is an injection. Indeed, say that $J_{x y}(u)=J_{x y}\left(u^{\prime}\right)=\left(d,\left(w_{1}, w_{2}, w_{3}, w_{4}\right)\right)$. Then, we have that $u=\left(w_{1} \oplus \boldsymbol{e}_{x_{1}}, w_{2} \oplus \boldsymbol{e}_{x_{2}}, w_{3} \oplus \boldsymbol{e}_{y_{1}}, w_{4} \oplus \boldsymbol{e}_{y_{2}}\right)=u^{\prime}$. It follows that $J_{x y}$ is injective. Second, we claim that $J_{x y}$ is a surjection on $S_{f(x, y)}$. Towards this end, we show that any tuple $\left(d,\left(w_{1}, w_{2}, w_{3}, w_{4}\right)\right)$ from $S_{f(x, y)}$ has a preimage $\left(u_{1}, u_{2}, u_{3}, u_{4}\right)$ under $J_{x y}$. Indeed, define $u_{1}=w_{1} \oplus \boldsymbol{e}_{x_{1}}, u_{2}=w_{2} \oplus \boldsymbol{e}_{x_{2}}, u_{3}=w_{3} \oplus \boldsymbol{e}_{y_{1}}$, and $u_{4}=w_{4} \oplus \boldsymbol{e}_{y_{2}}$. By construction, we have that $u$ is a preimage of $\left(d,\left(w_{1}, w_{2}, w_{3}, w_{4}\right)\right)$.

Finally, we observe that $\left(F_{1}, F_{2}\right)$ can be viewed as an encoding of $J$ since it encodes the $C(x, y, u)$ part by the pPSM $\left(H_{1}, H_{2}\right)$ and outputs $\left(u_{1}^{1}, u_{2}^{1}, u_{3}^{1}, u_{4}^{1}\right)$ as is. It follows, by standard properties of pPSM [AIK06, Lemmas 4.10 and 4.11], that the encoding $\left(F_{1}, F_{2}\right)$ is also a pPSM of $f$. Let $s_{F}, s_{J}, s_{H}$ (resp., $\left.t_{F}, t_{J}, t_{H}\right)$ denote the randomness complexities (resp., communication complexities) of the encodings $\left(F_{1}, F_{2}\right), J$, and $\left(H_{1}, H_{2}\right)$. By construction, we have that $s_{J}$ and $t_{J}$ are in $O\left(N^{1 / 2}\right)$ and, by Lemma B.2, we also have that $s_{H}$ and $t_{H}$ are in $O\left(N^{1 / 2}\right)$. Since $s_{F}=s_{J}+s_{H}$ and $t_{F}=t_{J}+t_{H}$ we conclude that $F$ has a communication and randomness complexity of $O\left(N^{1 / 2}\right)$. 